Foundations and Trends ${ }^{\circledR}$ in

Human-Computer Interaction

Vol. 2, No. 1 (2008) 1-93

(C) 2009 G. Johnson, M. D. Gross, J. Hong and

E. Yi-Luen Do

DOI: $10.1561 / 1100000013$

\title{
Computational Support for Sketching in Design: A Review
}

\author{
Gabe Johnson ${ }^{1}$, Mark D. Gross ${ }^{2}$, \\ Jason Hong ${ }^{3}$ and Ellen Yi-Luen Do ${ }^{4}$
}

1 Carnegie Mellon University, USA, johnsogg@cmu.edu

2 Carnegie Mellon University, USA, mdgross@cmu.edu

3 Carnegie Mellon University, USA, jasonh@cs.cmu.edu

4 Georgia Institute of Technology, USA, ellendo@gatech.edu

\begin{abstract}
Computational support for sketching is an exciting research area at the intersection of design research, human-computer interaction, and artificial intelligence. Despite the prevalence of software tools, most designers begin their work with physical sketches. Modern computational tools largely treat design as a linear process beginning with a specific problem and ending with a specific solution. Sketch-based design tools offer another approach that may fit design practice better. This review surveys literature related to such tools. First, we describe the practical basis of sketching - why people sketch, what significance it has in design and problem solving, and the cognitive activities it supports. Second, we survey computational support for sketching, including methods for performing sketch recognition and managing ambiguity, techniques for modeling recognizable elements, and humancomputer interaction techniques for working with sketches. Last, we propose challenges and opportunities for future advances in this field.
\end{abstract}




\section{1}

\section{Introduction}

People often sketch when solving problems. Some sketches are personal; others are collaborative. Some sketches help people make quick calculations and are quickly forgotten; others serve longer-term purposes. For professional designers, sketching serves as a means for thinking about problems as much as it does for communicating proposed solutions. For people who are not designers, sketching is a natural means for quickly recording spatial information such as directions to a point of interest.

Design can be seen as an iterative process of problem-framing and exploring possible solutions within the current conception of the problem. Sketching allows people to visually represent ideas quickly, without prematurely committing to decisions. A sketch is not a contract: it is a proposal that can be modified, erased, built upon. The rough look of hand-made sketches suggests their provisional nature.

Some theories of cognition give the human mind two distinct tasks: to perceive the world via our senses, and to reason about what our senses provide. In contrast, the late psychologist Rudolf Arnheim argues that perception and thinking are inseparable: "Unless the stuff of the senses remains present the mind has nothing to think with" [11]. Visual thinking is valuable in evaluating what is and designing what might be. 
Sketching allows people to give form to notions that are otherwise imaginary; the act of seeing fuels the process of reasoning.

The term "sketch" is used in many ways in vernacular and academic work. Some speak of sketching as a process - we sketch out an idea by talking about it, drawing pictures, or play-acting while considering possible solutions or problem formulations. Alternately we may use the term "sketch" to mean the product of an exploration, as when we make a prototype out of modeling clay, cardboard, or code.

In this survey, we define a sketch based on the utility hand-made drawings afford: sketches are quickly made depictions that facilitate visual thinking. In this way, sketches may include everything from doodles to roughly drawn circuit diagrams to an architect's quick isometric projection. We restrict neither the drawing medium nor the subject matter. Sketches are most often two-dimensional graphic depictions, but often incorporate textual annotations.

Sketching has been a topic of interest to computer scientists and HCI practitioners for quite some time. Early efforts such as Sketchpad [161] and GRAIL [39] hinted at the potential of pen-based interfaces. In fact, many of today's sketch-related research challenges were suggested by these systems 45 years ago.

Recently there has been a recurrence of interest in supporting sketching with computation. Computers can recognize user input and let people interact with drawings in ways that are impossible with paper alone, augmenting the sketching process in various ways. A rough sketch may contain enough information to infer the user's intentions. The drawing could then come alive, for example providing a simulation. Alternately the user's sketch may serve as a search query. Beyond recognition, a computer can render, rectify, or beautify a user's sketchy input into some other representation. Computation also supports editing operations that are impossible with physical sketches, for example, enabling collaborators in different locations to share an electronic drawing surface.

Researchers from many disciplines have contributed to knowledge about sketching and computational techniques for supporting it. Their diversity makes it difficult to get a complete sense of what has been done on this topic. This review draws from journals, conference proceedings, 
symposia and workshops in human-computer interaction, cognitive science, design research, computer science, artificial intelligence, and engineering design. These fields certainly overlap; however research in sketching lacks a unifying publication venue.

Some who study sketching as an element of design practice published in the Design Studies journal. Sketching has become a recurring theme at HCI conferences like CHI, UIST, IUI, and AVI, and visual languages conferences such as IEEE's VL and VL/HCC. The Association for the Advancement of Artificial Intelligence (AAAI) held symposia on diagrammatic representation and reasoning [49] and sketch understanding. The community brought together by the AAAI sketch understanding symposia continues meeting at the annual Eurographics Sketch-Based Interaction and Modeling workshop (SBIM). Related work has been published in computer graphics venues such as Computers and Graphics and the NonPhotorealistic Animation and Rendering conference. There is also a substantial amount of work published in various journals for electrical, mechanical, or software engineering.

Surprisingly, few surveys on sketch recognition and interaction have been published. Readers interested in pen computing in general may find Meyers' earlier review helpful [109]. That survey covers pen-related hardware, handwriting recognition, and presents a brief history of the traditional and computational use of pens but only briefly mentions sketching. Ward has compiled an online annotated bibliography of pen computing references that spans most of the 20th century [176].

\subsection{A Brief History of Pen and Sketching Systems}

Sketchpad was the first to demonstrate many techniques in computer science, human-computer interaction, and computational design [161]. It was an interactive design system allowing an engineer to create models by drawing with a light pen on a graphical display. The user could apply constraints (such as "make this line parallel to that line and maintain that relation") that relieved the burden of manually maintaining such relations. Figure 1.1 shows the user defining the shape of a rivet through a combination of drawing and constraint specification. 
RAND's GRAIL system (GRAphical Input Language) interpreted stylus input in a particular visual programming language for creating control sequence flowcharts [39]. GRAIL allowed users to quickly specify these programs graphically, rather than textually. To provide input, users drew or wrote freely on a digitizing tablet. GRAIL then attempted recognition using domain and contextual information to determine what the input meant (see Figures 1.2 and 1.3). The user could add semantically meaningful model data (boxes, arrows, writing) and issue commands (erase a line, move a box, change the data type of a node) without explicitly entering a mode.

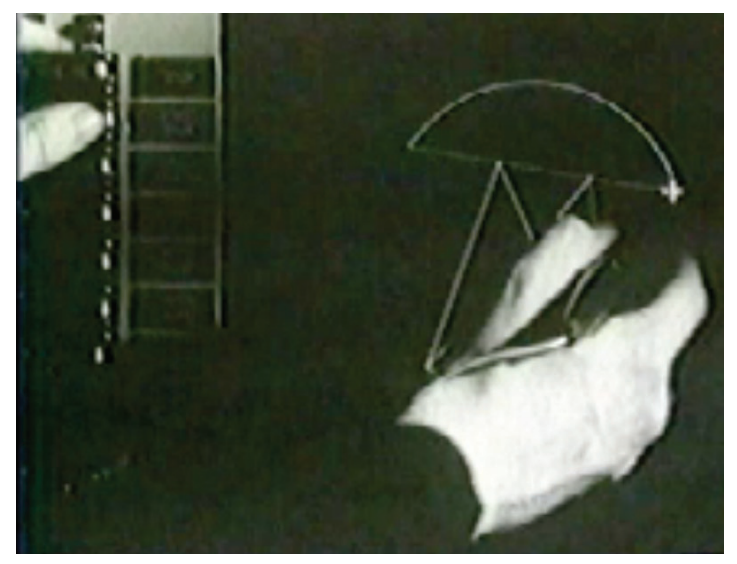

Fig. 1.1 Sketchpad supported users in creating design drawings using pen input (right hand) and constraints (specified by buttons aligned vertically at left).
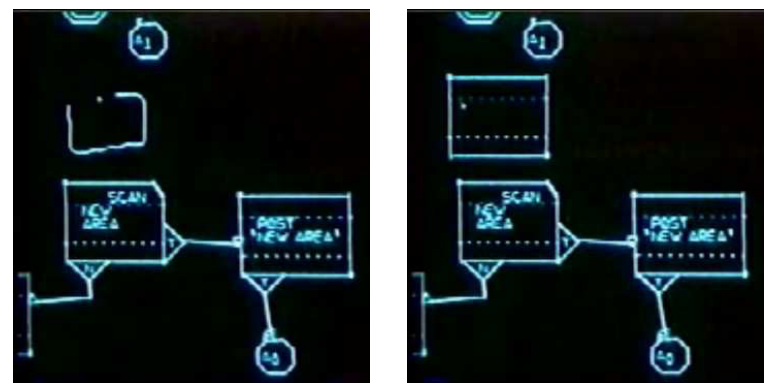

Fig. 1.2 On left, a GRAIL user draws a model element in place. At right, the rectified element is displayed as a box. 

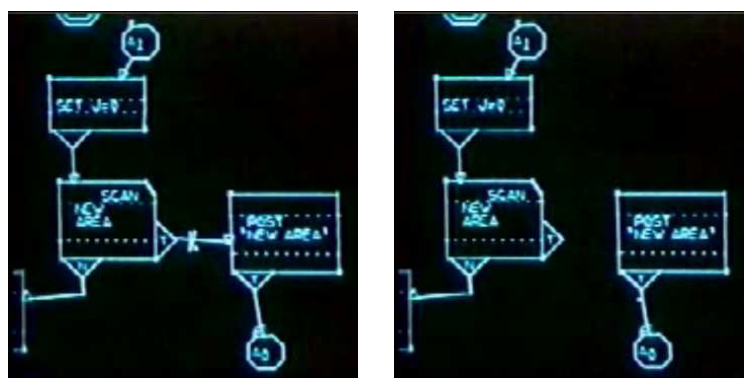

Fig. 1.3 GRAIL's sketch interpretation is context sensitive. At left the user crosses out the connector, which is interpreted as a delete command, shown at right.

Alan Kay discussed Sketchpad and GRAIL in a 1986 lecture. A portion of that talk is available on the Internet as part of the New Media Reader [83, 177]. Kay shows the video of these pioneering systems and provides insightful commentary, reminding viewers that much of the work in computer support for sketching has roots from several decades ago.

There was no widely used pointing device until the Macintosh brought about the mouse's widespread adoption in the mid 1980s. Owing to the success of the mouse, pen and sketch-based interaction was largely ignored for years. This began to change when commercial pen computing products came to market in the early 1990s, bolstered by the prospect of interaction based on handwriting recognition. Companies such as GO and GRiD developed and sold pen-based tablet devices. IBM's early ThinkPad computers (700T and 710T) were tablets. Yet these products fared poorly, and by 1995 many pen computing ventures had gone out of business. Pen computing did find a niche in the personal digital assistant (PDA) market with devices such as the Apple Newton and subsequently the more popular Palm Pilot. However, today's PDAs typically favor on-screen keyboards over stylus input. Tablet PCs are currently gaining popularity, primarily for making hand-written notes.

\subsection{Sketching Challenges in $\mathrm{HCl}$}

The strength of sketching input lies in the speed and fluidity with which people can express, interpret, and modify shapes and relationships 
among drawn elements without necessarily attending to details such as alignment or precise measurement. These strengths can also be seen as the weaknesses of sketching. The equivocal, imprecise nature of freehand drawing that so benefits humans is exactly why machines have difficulty recognizing sketches.

Those who aim to create useful and usable systems based on sketch recognition face a set of challenges including:

- Make hardware to support pen-based interaction.

- Build comprehensive, robus toolkits for building sketch-based systems.

- Create robust sketch recognition algorithms.

- Develop user friendly methods for training and modeling recognizable content.

- Design better interaction techniques for sketch-based systems.

This review elaborates on each of these challenges. Progress in one area will likely require simultaneous work in others. For example, in order to fully explore interaction design issues in recognition-based interfaces, we first need sufficiently robust and accurate sketch recognizers. In order to build recognizers capable of interpreting sketches made by any person in any domain we must have methods for modeling domain content. This in turn requires appropriate hardware and interaction methods.

\subsection{Research Themes in Sketch-Based Interaction}

This review details the primary themes of research shown in Figure 1.4: support for design, hardware, sketch recognition, and human-computer interaction techniques.

Traditional sketching: (Section 2) Sketching plays a crucial role in the practice of design. Sketching helps designers think about problems and offers an inexpensive but effective way to communicate ideas to others. The practice of sketching is nearly ubiquitous: One recent study of interaction designers and HCI practitioners found that $97 \%$ of those surveyed began projects by sketching [116]. We must understand the purpose and practice of sketching as it is done without computation if 


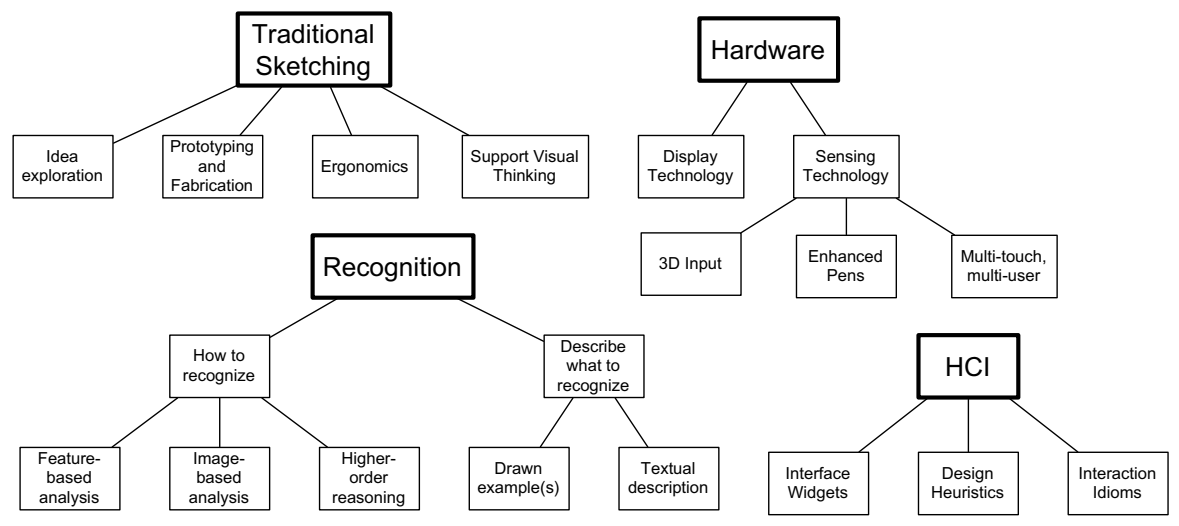

Fig. 1.4 Research themes for sketch-based interaction in design.

we hope to effectively support it with computation. Most research in computational support for design sketching has focused on the early phases when designers are exploring high-level ideas. Fewer sketchbased design systems support later stages of design when decisions must be formalized. This section provides a basis for thinking about how, why, and when (and when not) we may augment sketching with computation. This discussion covers the cognitive affordances of sketches and describes several empirical studies.

Hardware: (Section 3) Physical devices supporting pen-based input have existed since RAND's digitizing tablet was developed in the 1950s. Sensing technology (input) comes in many forms. Sutherland's Sketchpad system in the early 1960s accepted input from a light pen [161]. Some devices promote using fingers rather than pens, trading accuracy for convenience. Pen-based devices range in size from small (such as PDAs or "pentop computers") to medium (Tablet PCs) to large (electronic whiteboards). Other hardware considered by sketching researchers includes electronic paper and ink. The device's size and means for providing input dictate how and where it may be used, and how mobile it is. New kinds of devices will lead to new ways of interaction.

Sketch recognition: (Section 4) Recognition is central to many research systems in sketching. For this reason, a large portion of this 
review is allocated to discussing sketch recognition. Some drawn marks indicate domain elements, others should be taken as commands, while others are annotations. As with other recognition-based modes of interaction such as speech, sketch-based systems must have a model of what is to be recognized, as well as algorithms for performing that recognition. Some recognition techniques rely on input features such as corners, lines, and pen speed. Other techniques compare the image formed by user input with known elements. Still other techniques use artificial intelligence methods such as Bayesian Networks for reasoning about likely sketch interpretations. To recognize input the system must first have a model of what may be recognized. Models are frequently made by drawing examples. Other useful modeling strategies involve textual languages describing the shape and relationships among visual elements.

Human-computer interaction: (Section 5) User interfaces based on recognizing human speech, gestures, and sketching pose interesting challenges for researchers in human-computer interaction. New sketching input hardware, for example, may promote new interaction styles or allow people to interact with computers in new contexts, or collaborate in new ways. Because sketch input may be ambiguous, the interface should not necessarily treat it in the discrete, deterministic way that mouse and keyboard input is treated. Further, resolving ambiguity may be delegated to the user, which requires good interaction design. 


\section{Traditional Sketching}

Sketching is the traditional method for early-phase design when both problems and solutions are unclear. Whereas some might view design as a process that begins with a fixed problem definition and progresses by optimization within constraints to refine the problem solution, to others, the practice of design is as much a matter of defining the problem as solving it.

Sketching enables people to work with abstract and uncertain elements, interpreting and re-interpreting marks on the page that may be ambiguous or hold alternative contending semantics. Sketched figures, pictures, and maps help people to focus their thoughts or to explain ideas to others [36].

We talk about different kinds of renderings: artistic sketches, study sketches, drawings, diagrams, schematics, blueprints, and so on. Although the boundaries are not always clear, each is characterized by the means of production, the kind of information it communicates, and the role it plays in problem-solving.

Sketches support quick, informal information processing. We may write a phone number on the whiteboard near our desk rather than typing. Or, we may choose to make quick calculations or "To-Do" lists 


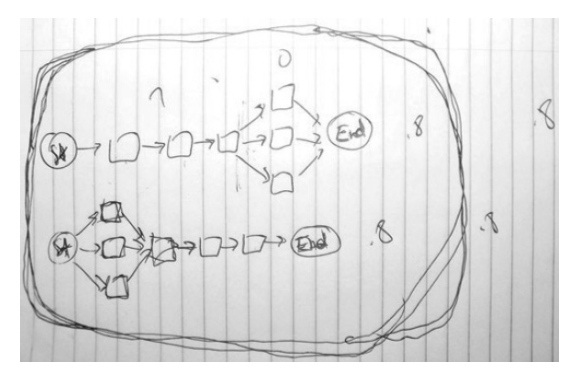

(a) Project management diagram showing task precedence of two projects. Hastily drawn boxes and arrows represent abstract activities.

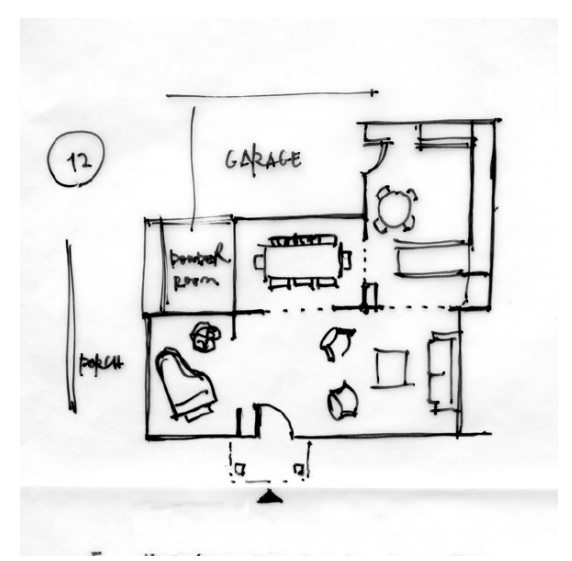

(b) An architect's floor plan sketch. It includes text, spatial information, and symbols representing household items like a piano or dining table.

Fig. 2.1 Sketches vary in domain and in the visual characteristics of marks.

on a sheet of paper because they are portable and easy to make. In his book How to Solve It, George Polya recommends drawing figures to help understand and solve problems in mathematics [134]. Sketches help us to visualize and restructure problems to make solutions more readily apparent. For example, the drawings in Figure 2.1 were made by practitioners in different domains. Each depiction helps designers to think about problems or possible solutions.

There is still a gap between what we know about how and why people sketch and what kinds of things we can do to support it computationally. To provide a basis for discussing computational support we first survey the design and cognitive aspects of traditional sketching done with physical media.

\subsection{Sketching in Design}

While designing, we iteratively explore and refine the problem definition and proposed solutions. Sketching supports this creative search process. We set out on our design task with some high-level goals. However, due to the ill-structured [157] and "wicked" nature of design [137], 
we encounter unforeseen opportunities and constraints as designing progresses. Those opportunities and constraints may be implicit in the original problem description, but designers expose them as they explore. The discoveries are incorporated into the understanding of the problem and potential solutions. Design problems are "not the sort of problems or puzzles that provide all the necessary and sufficient information for their solution [28]." So it goes with sketching. We draw different views of our model, which allows us to perceive the problem in new ways.

Designers engage in a sort of "conversation" with their sketches in a tight cycle of drawing, understanding, and interpreting [148]. Goldschmidt describes this as switching between two reasoning modes: "seeing that" and "seeing as" [51]. Seeing that is the process of recognizing the literal, descriptive properties of the drawing. Seeing as is figurative and transformative, allowing the designer to re-interpret parts of the sketch in different ways. For example, a designer may draw a sofa and see that the wood constituting the back rest and the rear supports come into contact. In this way, the designer can see how the two parts could be redesigned as one. The designer may be able to extract more information from the sketch than is consciously put into it [52].

Care must be taken to support this reflection when making design software that employs sketch recognition. If the system interprets drawings too aggressively or at the wrong time, it may prevent the human designer from seeing alternative meanings; recognize too little and the software is no better than paper. This is discussed further in Sections 4.1 and 4.3 .

Expertise plays a role in sketching as well. Design students begin to sketch from the first day. Proficiency in sketching goes beyond applying marks to the page - one must also interpret and re-interpret drawings in order to use them effectively. Suwa and Tversky studied differences between student and professional architects, finding that professionals had greater skill in transformative reasoning [162]. Buxton points out the importance of recognizing the disparity between sketchers who can expertly see as and see that, and those who can not [21, p. 118]. Trained designers skillfully examine the content from multiple perspectives, extracting different interpretations. 
Experimental and observational evidence suggest that people of comparable skill use consistent methods when making drawings. This consistency applies to the motor action of drawing [171] and to higherlevel semantic notation. For example, the type of an architectural drawing (e.g. floor plan, elevation, bubble diagram) can be discerned by identifying particular visual elements the designer employs [36, 121].

\subsection{Prototyping and Fidelity}

Newman and Landay conducted ethnographies of web designers, focusing on the use of informal design techniques [122]. The designers were observed making models in various media (such as on paper or with a computer) at various levels of fidelity. Designers always sketch at the beginning of a web design project, exploring numerous highlevel options. Frequently this early sketching phase is accompanied with construction of low-fidelity prototypes made on paper or with Microsoft PowerPoint ${ }^{\mathrm{TM}}$ [12]. As the design progresses and designers begin making incremental edits, they move to higher fidelity models. Client meetings are an important forcing function in web design projects. When meeting with clients, designers want to show polished prototypes produced with computer software. Therefore, designers used electronic tools earlier in the process than they would otherwise have preferred.

Today, most software tools support incremental refinement and specification of details but do not adequately support idea generation or exploration [163]. Designers who begin using software tools in the early phases of design tend to make superficial explorations of possible solutions. Further, because tools are poor for exploration but good for specifying details (font, line weight, and color), designers tend to focus on nuances that are not yet important. Observing that current tools are inadequate for creative pursuits, researchers have developed calligraphic tools such as SILK and DENIM, which aim to support the early phases of design $[92,99]$.

Paper sketches dominate the early phases of design as people generate new ideas, in a process Goel terms "lateral transformations" [50]. But as soon as the web designer believes he or she will make incremental 


\section{Traditional Sketching}

revisions (which Goel calls "vertical transformations") they switch to a computer tool.

It is not always best to use high-fidelity models for testing designs. Walker and Takayama observed web site developers performing usability tests of ongoing interface designs. They found that high-fidelity, computer-based models are not significantly better than low-fidelity, paper-based models when testing design ideas [174]. This finding supports the conventional wisdom that inexpensive and quickly made lowfidelity prototypes are appropriate in iterative design, building, and testing.

\subsection{Sketches as a Symbol System}

Goodman provides a comprehensive framework for analyzing the properties of various symbol systems, including sketches [53]. Goel places sketching in Goodman's framework, noting that sketches have overloaded semantics, they are ambiguous, dense, and replete [50]. These properties describe one particular sense of sketching in which the drawer's marks may be idiosyncratic.

Sketches have "overloaded semantics": The same symbol may mean different things depending on context. Further, a sketched symbol may be "ambiguous," meaning that the symbol affords more than one plausible interpretation. Figure 2.2 illustrates these properties. A lumpy shape can be used to indicate many things including clouds, trees,

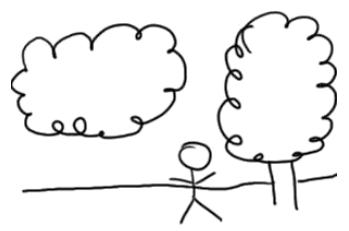

(a) Overloaded semantics: The cloud and tree have similar shapes but different meanings due to context.

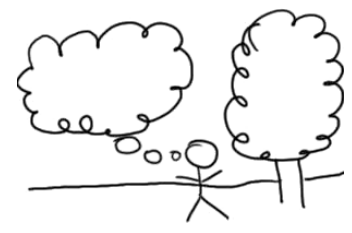

(b) Ambiguity: Changing the drawing slightly changes our interpretation. The object on the left may be a cloud, or it may be a cartoon thought bubble.

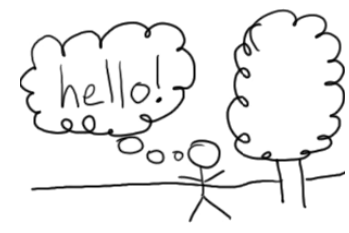

(c) Additional information helps us reason about the intended identity of elements. Text inside the cloud indicates it is a cartoon thought bubble.

Fig. 2.2 Overloaded semantics and ambiguity. 
or thought bubbles. We interpret the shape differently depending on context.

Sketched symbols are "dense," indicating there is a continuous range between instances of the same symbol. While there may be minute visual discrepancies between symbol instances, Goel claims that such symbols are also "replete": no aspect of the sketched symbol may be safely ignored (Figure 2.3).

The pen strokes constituting a sketch serve various functions. Ink may indicate abstract domain symbols (e.g. diode, treble clef), object boundaries, actions (e.g. arrows indicating containment or movement), dimensions and units, annotations, region texturing, and so on. Some parts of a sketch are more dense and replete than others. For example, a diode's properties do not change if it is drawn with a slightly larger triangle. However, subtle variations in how a desk lamp is drawn might lead to substantially different aesthetic responses to it.

Gross and Do discuss some properties of hand-drawn diagrams from the perspective of building tools to support design drawing activities [59]. The authors distinguish sketches from diagrams, noting that diagrams are "composed of primitive elements chosen from a small universe of simple symbols - boxes, circles, blobs, lines, arrows." This list is certainly not exhaustive, but it does illustrate the general idea that diagrams have a limited vocabulary. In practice, sketches and diagrams from various dialects may be combined (e.g. mathematical notation on the same page as circuit diagrams and hand written notes).

Freehand diagrammatic drawings are abstract, ambiguous, and imprecise. Abstract symbols denote elements whose identities or properties are not (yet) important or known. For example, Figure 2.1(a) shows a project management diagram of two hypothetical projects. The

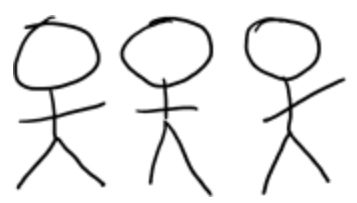

Fig. 2.3 Different instances of the same stick figure vary along a continuum (dense). However, the visual properties of individual symbols may (or may not) communicate additional information (replete). Is the figure at the right waving? 
activities composing each project are abstract - they could represent anything. The value of the sketch is that it shows the project's network topology and does not draw attention to what the specific activities are.

An ambiguous symbol has many plausible interpretations. The floor plan sketch in Figure 2.1(b) shows several rectangles indicating rooms, furniture, shelves, or counters. Human observers can confidently disambiguate the intended meaning of some rectangles, but others remain unclear. The bottom-right of the sketch shows two armchairs and a sofa with an ambiguous rectangle in the middle that could plausibly represent either a rug or a coffee table.

Last, freehand diagrams are imprecise. Imprecision allows designers to work with rough values (e.g. "about two meters wide") and avoid premature commitment. Imprecision also indicates that the design is by no means final.

The notational properties of sketches make them powerful tools for supporting visual thinking. Designers may leverage ambiguities in their sketches to see new meanings, for example. However, these same properties present challenges for accurate software recognition.

The degree to which a drawing is ambiguous, imprecise, and abstract varies among instances, and people might interpret them differently. A rough sketch is useful to designers, especially for brainstorming and incremental development of ideas. But in order for the sketch to be transformed into a finished product (e.g. for manufacturing), it must be made unequivocal, precise, and concrete. The process of moving from the informal sketch to the formal specification involves drawings that are semi-ambiguous, partially precise, and with some abstractions given definite identities.

\subsection{Cognitive and Mechanical Aspects of Drawing}

Larkin and Simon compared diagrammatic notation with sentential (written or spoken) systems, suggesting that spatial notation often affords more efficient information processing than an equivalent written statement [93]. Efficiency of information processing refers to how much computation is necessary to translate input into understanding. For example, we may state the relationship between supply and demand in 


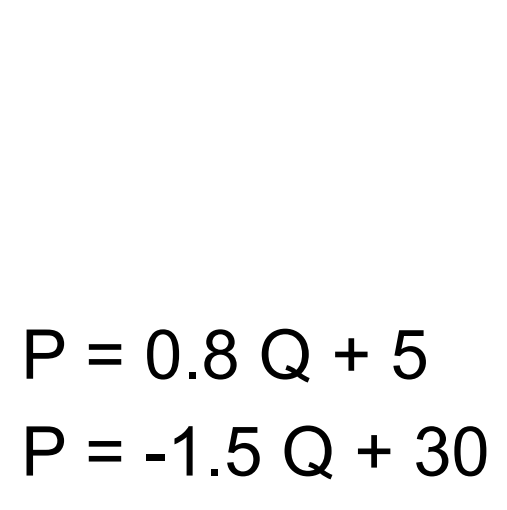

(a) Written (sentential) form.

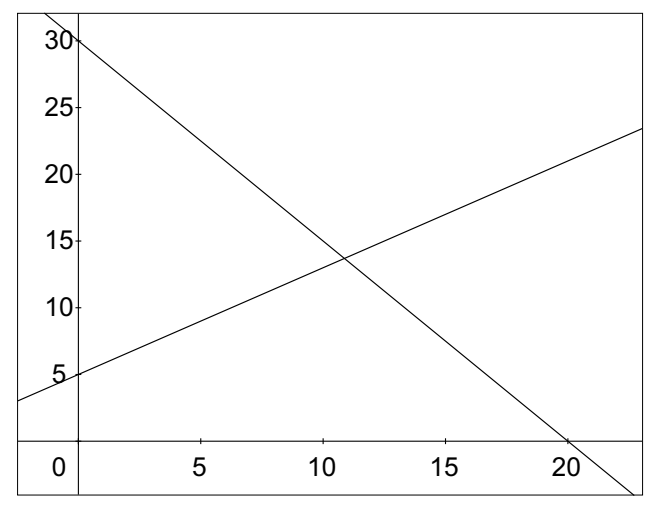

(b) Graphic (diagrammatic) form.

Fig. 2.4 Sentential and diagrammatic representations of supply and demand provide equivalent information.

a market economy with a mathematical (sentential) description as in Figure 2.4(a). Market equilibrium is found where the values of supply and demand intersect. In a sentential representation, we must calculate to find equilibrium. However, given a graph (diagrammatic) as in Figure 2.4(b), we directly see where the supply and demand curves intersect and immediately read the associated values. Even though the representations provide equivalent information, the diagrammatic form can be more efficient, depending on the viewer's goals.

When people sketch, they "incorporate relevant information and omit the irrelevant" [168]. Drawing allows people to use paper as external storage, reducing and augmenting cognitive load. Diagrammatic notation schematizes domain information [170]. Hand-drawn route maps, for example, are usually made with a simple visual vocabulary consisting of rectangles or circles indicating buildings, lines or arcs representing paths, and junctions where paths intersect [169]. The shape and curvature of buildings and paths is drawn approximately. Given a well-drawn map, humans have little trouble reconciling differences between the map and the physical world it represents.

Spatial representations offer a powerful means for learning in many domains. Sketching allows learners to represent concepts as they understand them, and allows more experienced people to spot inconsistencies or areas where additional learning is needed [42]. 
Van Sommers extensively studied how people draw [171]. This includes the mechanics of using one's arm, wrist, hand, and fingers when drawing. His research examines the effects of culture, handedness, and expertise on how drawings are made. He found that of the many possible ways of making a drawing, subjects tended to use consistent strategies. For example, subjects were asked to trace over short lines scattered about a large sheet of paper. The lines did not overlap and represented the full range of rotations. Right-handed drawers had a strong tendency to draw from left to right and top to bottom. Lefthanders also showed the tendency to draw top to bottom but showed a slight preference toward drawing right to left.

Sezgin gives a compelling example of consistent stroke ordering [151]. Participants were asked to draw common objects such as the stick figure shown in Figure 2.5 thirty or more times. This stick figure has six components: the head, a torso, two arms, two legs. The lines can be drawn in 720 distinct sequences $(6 !=720)$. However, participants used only about five of those component orderings to draw the figure. Further, each participant showed a strong individual bias to draw the symbol using the same sequence (e.g. head, torso, legs, and finally the arms).

A drawer's preferred direction of making strokes depends on the semantics of whatever is being drawn. Van Sommers shows that although people will choose to draw arbitrary lines according to a preference (largely determined by handedness), people will deviate from

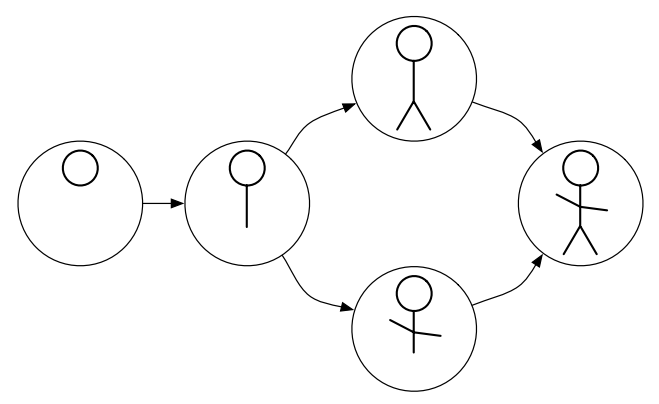

Fig. 2.5 A stick figure with six components can be drawn in any one of 720 possible orders, but only about five are used in practice [151]. Two of those orders are shown in this sketching style diagram. 
those preferences when drawing things that hold meaning. Participants were asked to draw common things, including a rake and cigarette smoke. We know from experience that a rake is held at the top with the rake's fingers contacting the ground. Cigarette smoke rises. Van Sommers' participants generally drew the rake from top to bottom, but the smoke from bottom to top. He posits this is because our knowledge of an object's properties informs the way we draw it.

Stroke ordering can also be affected by perceptual properties of a drawing, even if the drawing's meaning is not clear. Van Sommers asked experiment participants to trace over the picture in Figure 2.6. Each "grape" in a cluster has a boundary. The number represents the mean position in the drawing sequence. The grape "on top" is usually drawn first, with neighboring grapes drawn next.

Van Sommers uses the term anchoring to explain the sequence that some strokes are made. Anchoring is an example of a control strategy for making marks. The grape "on top" serves as a convenient anchor for drawing its neighbors. It is easier to control the start and end locations of a mark if there is already something to attach it to. Another control strategy for making marks is containment. In depictions with a structure enclosing something else (such as a bowl of fruit), the container is

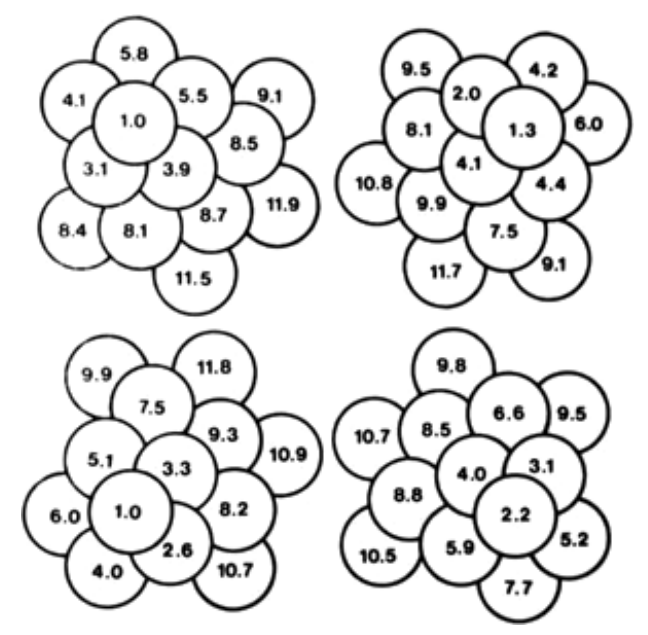

Fig. 2.6 "Grape Clusters" drawn by right-handed participants in van Sommers' study showing the mean position in the drawing sequence. 
typically drawn first. Anchoring and containment may be useful control strategies for improving sketch recognition efficiency.

Some researchers have noted the prominent place of sketching in design and have studied sketching as a tool. A series of experiments by Goel looked at the cognitive affordances of sketching [50]. These studies compared traditional pencil-and-paper sketching with sketches made with a computer drawing application. Participating designers were asked to design an artifact using either a software paint program or using pencil and paper. The software tool was modified to only support structured input such as straight lines, rectangles, and ellipses. He found that the structured notation system (computer application) did not afford the same level of rapid exploration of design ideas as using an unstructured system (pencil-and-paper freehand sketching). Sketching supports designers in easily trying many ideas, which Goel calls lateral transformations. This is distinguished from vertical transformations, characterized by refining ideas rather than exploring new ones.

The transformation types identified by Goel correspond to the activities done at different stages of design described by Newman and Landay [122]. Early in the design process designers are concerned with idea generation and exploration (lateral transformations). Later on designers make iterative revisions and refinement (vertical transformations). The goal of many computational sketching tools is to support lateral transformations.

\subsection{Summary: Traditional Sketching and Computation}

If we hope to effectively support sketching with computation, we must first understand the practical aspects of traditional sketching.

Sketching is an important - perhaps necessary - tool for doing design. It gives us a way to quickly make provisional drawings, which help us efficiently make sense of spatial, relational information. Sketches let us make marks that are as vague or specific as we need. Because sketched elements can easily be ambiguous, rough drawings afford different interpretations. We may therefore reflect on our sketches and see new meaning in existing marks. People sketch in part because they 
do not know exactly what they are making — sketching facilitates exploration.

Low-fidelity prototypes are especially important as tools to test ideas during early design. This is because they are easy to make, allowing designers to quickly expose problems before committing to decisions. Sketching is a common method of creating such prototypes.

In order for computers to recognize sketches, we must develop techniques to transform imprecisely made marks into discrete symbols. However, some of the properties that make a sketch useful for a human (overloaded semantics, ambiguous, imprecise, etc.) complicate the task of computer recognition.

We can make use of consistencies in how people draw to inform recognition. First, we observe that some kinds of sketches are made using restricted visual vocabulary, e.g., diagrams of electronic circuits or web page layouts. Second, consistencies in the mechanical act of drawing (such as anchoring and containment) can be used to further guide recognition. For example, recalling Figure 2.5 we know that people are likely to use only a small number of ways to draw a stick figure even though there are hundreds of possible stroke orderings. This can help make recognition strategies more efficient by utilizing commonly used patterns before less frequently used ones. 


\section{Hardware Support for Sketching}

Today's pen hardware is largely oriented toward reading and writing text. However, when considering hardware support for design activities, we may compare current computational hardware with the physical artifacts designers use in practice. Designers can quickly draw on sheets of paper, and flip through them easily. Or we might pin the papers next to each other on a wall to compare and contrast ideas. The tip of the pen instantly deposits as we draw exactly where we have made contact with the page. We can use two hands to rotate the paper to a desired angle or use secondary devices such as a straight edge in aid of careful drawing.

It would be simplistic to argue that computational support for sketching should exactly mimic practices supported by physical media. However, current hardware lacks the portability, responsiveness, and feel of tools of traditional design practice. Commercial development and research continue to improve hardware support in these categories.

Many devices such as PDAs, tablet-style computers, and wallsize interactive screens feature some kind of pen (stylus) or touch input. While pen-based systems have been available to researchers for decades, only since the mid-1990s have systems become inexpensive and common. 
Hardware for sketching can be separated into two groups: those that support input only, and those that support input and output.

Some digitizing tablets afford input by letting people write or draw with a stylus. These are not output devices - they do not display the user's marks. Sketches are also input by scanning drawings. For example, PARC's research system ScanScribe analyzes marks made with everyday pen and paper to produce a computationally enhanced version of the drawing [141]. It is common for a single device to provide both input and output where the drawing surface is the same as the display surface, as exemplified by Tablet PCs.

Here, we consider hardware that is appropriate for use in sketchbased interactive systems. This hardware ranges from small (pen computers, PDAs) to medium (tablets, desktop workstations) to large (table top or whiteboard systems).

\subsection{Computationally Enhanced Pens and Paper}

We begin our discussion of sketching hardware with commercially available devices such as the Anoto Pen [7]. They are slightly larger than ordinary pens, but small and light enough to use like any other pen. They mark the paper with ink, letting users keep a paper record of their use. Pen activity can be transmitted as it is used, or stored for later use.

The Anoto Pen is used on special paper featuring a pattern of tiny dots. Each sheet has a unique pattern. A small infrared camera monitors a region near the pen's tip. Firmware decodes the local pattern of dots and determines where the user is marking.

Device producers have licensed Anoto's technology and produced their own pens, such as Logitech's io2 or LeapFrog's Fly pen. The Fly recognizes a limited set of user actions and provides audio feedback with a small speaker housed inside the pen. For example, the user can draw a simple calculator on Anoto paper and then use it to perform basic arithmetic. The pen speaks the calculations it makes.

Paper Augmented Digital Documents (PADD) are a class of "digital documents which one can manipulate either electronically or on paper" [62]. It provides ways to bridge the gap between virtual and 
physical paper. For example, ModelCraft enables users to manipulate electronic 3D models using paper as the input device [158]. PaperPoint allows control and annotation of PowerPoint presentations using printed copies of slides [156]. West et al. describe their Anoto-based MEMENTO system that elders use to create digital scrapbooks using paper [178]. MEMENTO and PapierCraft and other augmented paper technologies leverage the convivial, easy-to-use aspects of pen-andpaper while enabling people to easily manipulate virtual artifacts.

Another enhanced ink pen device was the CrossPad, sold from 1998 to 2001 [30]. It allowed users to write on a traditional pad of paper using an ink pen. The pen's location was calculated by a receiver clamped to the writing pad. Ink data could later be transferred to a PC, which performed handwriting recognition.

\subsection{Input Surfaces and Styluses}

Many digitizing tablets sense pressure [109]. Designers often use heavier lines to indicate object boundaries, and lighter lines to indicate subtle (but potentially important) texturing, shading, or curvature. Devices that sense pressure can allow designers to create thicker or darker lines without changing drawing tool modes.

Pressure sensing has been implemented in a number of ways, for example, two conductive layers with current flowing in orthogonal directions. The layers do not touch and may be separated with a nonconductive layer of fluid. When something (a pen, a finger) contacts the upper surface, voltage changes are measured at layer edges. The contact location is calculated by interpolation. Other touch surfaces sense electrical properties of things contacting them — this is why a gloved finger will not work on many laptop trackpads. Wacom ${ }^{\mathrm{TM}}$ tablets and similar inductive devices depend on a special stylus that resonates in an electromagnetic field generated by the tablet surface. Still other tablets detect acoustic [33] or optical disturbances for calculating input position.

Some sensing surfaces report a single location of contact, but multitouch surfaces identify more than one input coordinate. Recent multitouch systems have become popular, including Han's Frustrated Total 
Internal Reflection technique [65] and Microsoft's Surface system [110]. MERL's DiamondTouch is a multi-touch tabletop display that also supports multi-user interaction. DiamondTouch discerns individual users based on different capacitance levels as the user completes a circuit between the surface and a pad placed on their chair.

Input surfaces that are intended to be used with fingers or hands offer different interaction experiences than pen-oriented drawing surfaces. For example, users may trace shapes with a single finger, use two fingers to zoom in or out, or use whole-hand gestures for issuing other commands. These interaction techniques may provide opportunities for developing innovative sketching applications.

\subsection{Distinction Between Pen and Mouse}

Regardless of sensing technology, all these devices allow users to provide input in a way that is much closer to traditional writing than a mouse allows. Although pen and mouse input share many properties (both allow users to interact with 2D displays) they have several key differences.

Mouse input affords motion sensing while pen input affords position sensing [67]. In other words, while mice produce the relative change in $(x, y)$ locations, pens directly provide absolute $(x, y)$ locations. Users can configure tablets to report relative position, thereby behaving like a mouse.

Form is also extremely important. A stylus affords people to use the fine motor abilities of their fingers to control the tip of the pen, whereas hand and forearm muscles dominate mouse usage. Fingers can be used to move the mouse, but not with the same dexterity possible with a pen. Depending on the type of work, a pen may be ergonomically superior to a mouse, or the other way around.

Digitizing tablets can detect more than the stylus position. Some devices sense stylus pressure, its angle relative to the tablet, or its rotation. The nonwriting end of the stylus is sometimes used as an alternate tool mode (e.g. as an eraser).

Some styluses have buttons. While buttons are an indispensable part of a mouse, they are often difficult to use on the barrel of a 

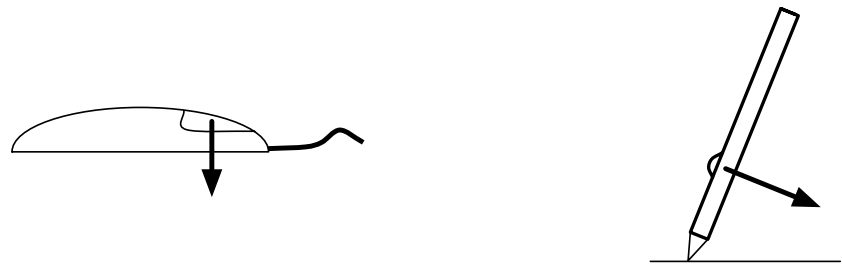

(a) Pressing a mouse button exerts force perpendicular to the operating plane.

(b) Pressing a stylus button is likely to cause accidental pen tip movement.

Fig. 3.1 The force required to press a mouse button compared with a stylus button.

pen [132]. The force of a mouse button click is orthogonal to the device's plane of use and has negligible effect on target accuracy. However, pressing buttons on a stylus can move the tip of the pen, making it difficult to press the button while pointing at particular objects (see Figure 3.1). Further, pressing a button on a computer stylus usually requires the user to adjust the pen in hand. This action may be distracting and uncomfortable for long term use.

\subsection{Large Displays for Drawing}

Groups of people often gather around traditional whiteboards to brainstorm or exchange ideas. They draw pictures and diagrams, maintain lists of text, and so on. Whiteboards are also effective for leaving asynchronous messages in the physical workspace where co-workers can see them. People sometimes photograph whiteboards or scan pages of sketches in order to record works-in-progress.

Electronic whiteboards such as the SmartBoard or those produced by PolyVision more readily afford interactive usage than ordinary whiteboards. For example, collaborative systems such as Colab [160] and Tivoli [131] support meetings as people maintain checklists or collaboratively draw. Systems can dynamically change their displays as people interact with them, resizing or moving objects, or checking items in a list [117]. Large displays let more people have simultaneous access to the drawing surface, which presents challenges in sensing, and distinguishing between different users' pens. 


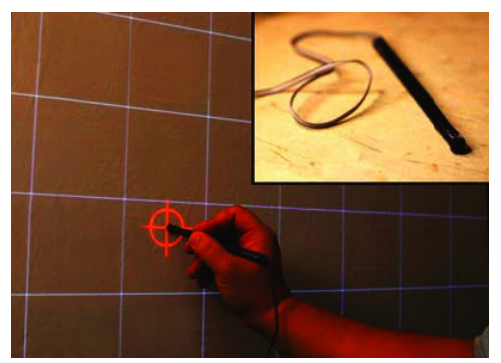

(a)

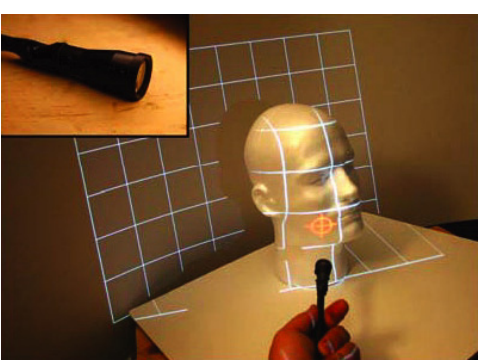

(b)

Fig. 3.2 Lee's portable projection approach enables detection of drawing surface size and orientation as well as pen input. This is effective on flat surfaces as in (a) or curved surfaces as in (b).

Large displays have historically lacked portability, requiring a good deal of time to install and calibrate. However, Lee recently demonstrated a portable approach for projecting images on large surfaces such as walls or tables, shown in Figure 3.2 [95]. The projector emits patterns of time-multiplexed visible and infrared light. The infrared light is decoded by sensors, providing a host computer with fast and accurate location detection. The system uses sensors embedded in the display surface to calculate the surface size and orientation relative to the projector. Sensors may also be embedded in objects such as pens, enabling alternate methods of user input. Equipping a pen with a focusing lens allows it to become a short-distance pointing device as in Figure 3.2(b) [96, p. 62].

At least two methods for capturing marks on ordinary whiteboards utilize physical ink-like markers. The first uses traditional markers whose activity can be detected by nearby sensors or cameras as the user works, such as the Mimio commercial system. Whiteboard markers are placed in sleeves whose position is calculated by the system hardware. Ju et al. used a Mimio in the WorkspaceNavigator [77]. PARC's ZombieBoard $[112,140]$ used the second method: scanning the whiteboard optically using a still camera. The ZombieBoard enabled users to interact with the computer by drawing special symbols. For example, users could delimit a region of the whiteboard to be scanned by drawing its boundary. To invoke the scan, the user would draw a button on the region boundary and draw an $X$ or check mark inside. 


\section{Sketch Recognition Techniques}

Just as speech and gesture recognition gives us powerful new interaction avenues, sketch recognition allows a different paradigm for interacting with computers. This section summarizes strategies for performing sketch recognition.

An important subset of sketch interpretation is handwriting and character recognition, which is responsible for converting a person's handwriting into text. Microsoft's Tablet PC API includes handwriting recognizers for several languages, as well as symbol and gesture recognizers for notation systems such as music or mathematics. The recognition supported by the Tablet PC and other commercial handwriting recognizers (e.g. Apple's Inkwell [10]) is more closely aligned with what Larkin and Simon refer to as "sentential" [93], as user input is expected to proceed in a linear, serial fashion. This contrasts with "diagrammatic" representations that are expressed over a 2D plane.

Modern pen-based computer systems like PDAs or Tablet PCs have handwriting recognition software that has usable accuracy rates. Recognition accuracy is typically measured by the deviation between what the user intended and the machine's interpretation. Error tolerance refers to the recognition inaccuracy rate a user is willing to accept. 
One study involving a modified typewriter found that typists do not notice a word $0.5 \%$ error rate, $1 \%$ is manageable, but $2 \%$ is unacceptable [25, p. 79]. It is unclear if there are similar accuracy thresholds for sketching, though studies have been conducted for related recognition tasks. For handwriting recognition, adults find 3\% minimally acceptable, with $1 \%$ considered "very good" [91]. The acceptable accuracy level depends a great deal on the expected benefit of the task. Users will accept $20 \%$ error rates if the tool brings greater productivity, but are intolerant of errors ${ }^{1}$ when the perceived benefit is smaller [44]. Another study examining user acceptance of hand gesture recognition error rates found users would tolerate mis-recognition up to $10 \%$ when equivalent keyboard commands could be employed [81].

There are a number of methods for improving recognition. Many PDAs recognize characters from a constrained symbols set (Latin letters, Arabic numerals), using different areas of the input surface for drawing numbers and letters. This approach is not natural, but many users quickly learn the alternate way of writing. In contrast, Microsoft's Tablet PC handwriting recognizer allows people to write in their own handwriting, at any location or angle. Microsoft's recognizer incorporates a language model (e.g. spelling, grammar) to guide interpretation.

Recognition is the centerpiece of many software prototypes that support sketching. Although the research focus of many systems is not recognition, such systems use automatic interpretation of sketches to explore new methods of interacting with computers and new ways of designing $[60,61,99]$. Such projects rely on reasonably accurate recognizers. Recognition is therefore a topic that affects nearly all aspects of research on sketch-based systems. For this reason, a substantial portion of this review is devoted to aspects of sketch recognition. This includes timing issues, determining what to recognize and how those elements can be recognized, and interaction issues (see Table 4.1).

${ }^{1}$ It is unclear if the values described in these articles measure character or word recognition error rates. Santos et al. describe handwriting recognition error rates of approximately $10 \%$ in terms of characters [139]. 
Table 4.1 Topics in sketch recognition.

\begin{tabular}{|c|c|c|}
\hline Topic & Section & Remark \\
\hline When to recognize & 4.1 & $\begin{array}{l}\text { Recognition is powerful but may also distract users } \\
\text { from their task. }\end{array}$ \\
\hline What to recognize & 4.2 & $\begin{array}{l}\text { Sketches may represent objects (e.g. tables and } \\
\text { chairs) and spatial or functional relationships } \\
\text { between those objects (e.g. chairs positioned } \\
\text { around table perimeter). }\end{array}$ \\
\hline $\begin{array}{l}\text { How much to } \\
\text { recognize }\end{array}$ & 4.3 & $\begin{array}{l}\text { Sometimes only a portion of a sketch need be } \\
\text { recognized. }\end{array}$ \\
\hline How to segment & 4.4 & $\begin{array}{l}\text { Sketches contain many different symbols that may } \\
\text { overlap. Recognizers must isolate groups of marks } \\
\text { for consideration. }\end{array}$ \\
\hline How to recognize & $4.5,4.6,4.7$ & $\begin{array}{l}\text { Many recognition techniques exist, and are reliant } \\
\text { on segmentation methods. }\end{array}$ \\
\hline $\begin{array}{l}\text { Training } \\
\quad \text { recognizers }\end{array}$ & 4.8 & $\begin{array}{l}\text { In order to recognize something we first need a } \\
\text { concept of whatever is to be recognized. }\end{array}$ \\
\hline $\begin{array}{l}\text { Managing } \\
\text { recognition error }\end{array}$ & 5.1 & $\begin{array}{l}\text { Recognition sometimes results in inaccurate, } \\
\text { ambiguous, or undesired results. }\end{array}$ \\
\hline $\begin{array}{l}\text { Reacting to } \\
\text { recognition }\end{array}$ & 5.2 & $\begin{array}{l}\text { Upon recognition the system must determine how to } \\
\text { respond: provide visual feedback, issue a search } \\
\text { query, or take no outward action. }\end{array}$ \\
\hline $\begin{array}{l}\text { Toolkits for } \\
\quad \text { recognition }\end{array}$ & 5.3 & $\begin{array}{l}\text { Software packages facilitate easier engineering of } \\
\text { new recognition systems. }\end{array}$ \\
\hline
\end{tabular}

\subsection{When to Invoke Recognition}

It is important that sketching systems aid design exploration and not simply computerize it [120]. For example, a sketch recognition system might zealously identify parts of the user's drawing as it is made, replacing the rough sketch with lines or curves. This removes the opportunity for reflection and re-interpretation that is so important to the early phases of design [51, 147, 163]. Premature recognition may interrupt the designer's flow of thought and interfere with the task at hand. Instead we may want the computer to eavesdrop silently, and provide help only when we need it.

A system can provide the user feedback of sketch recognition on different occasions: (1) immediately after receiving a single stroke of input, (2) as the user works, when feedback may be appropriate, (3) only upon request, or (4) never. Many interfaces attempt immediate recognition of gestures for invoking commands, such as with marking menus [87] or PDA device character input [127]. This is commonly called eager 
recognition [20]. Other systems $[4,59,72]$ perform recognition in the background, deferring judgment until adequate information is available. This is termed lazy recognition. Most modern research prototypes take this approach. Last, some systems wait until the user explicitly requests recognition [92], or avoid recognition entirely [43]. Combinations of these approaches are common.

One method for identifying sketch input is to sidestep the recognition process altogether and tell the system what you are about to draw by choosing from a list or pressing a button. Forbus and colleagues take this approach as they "engineer around the need for recognition" in their nuSketch Battlespace system (nSB) for managing courses of action for military commanders [43]. In order to position an object such as an armored unit or a movement path, the nSB user selects an item from a palette of glyphs and draws on the map. The system interprets the user's ink differently depending on which type of object is selected. For example when placing military units, the ink's centroid is taken as the intended location; when defining a region for those units to defend, the boundary is used.

\subsection{What Should be Recognized}

User input in sketch-based applications may be provided in two broad categories. The first category, often referred to as digital ink, includes stroke timing information. Time data might be used by sketching applications to determine how quickly the user was drawing, or when strokes were made relative to one another. Many recognition algorithms depend on the presence of time information. The second category describes user input for which time data is unavailable. This category of sketch input is used by applications that depend on scanned or photographed input. Section 4.5 describes the differences between these two categories of sketch input from the recognizer's perspective.

Different kinds of design drawings ("model ink") in many domains might be recognized: artistic sketches, study sketches, drawings, diagrams, schematics, blueprints, and so on. In addition to model ink comprising those drawing types, user input may be interpreted as a command ("command ink"). The particular rules about what should be 
recognized depends on the domain (architecture or circuit design), kind of model (floor plan or timing diagram), and other application-specific requirements. Sketches often contain hand-written labels or annotations, which are common targets of pen-based recognition.

Diagrams usually have a domain-specific grammar describing how vocabulary items relate [90]. For example, boxes may connect to other boxes via lines, those lines may have arrowheads to indicate direction. Diagrams are good candidates for recognition because the various elements and their relations can be described formally. Table 4.2 summarizes various recognizable classes.

Artistic sketches and those drawings meant to authentically convey three dimensional form comprise a different recognition challenge. Nealen et al. (who are concerned with modeling 3D form) suggest that "sketching a shape is inverse NPR (NonPhotorealistic Rendering)" [119]. Drawings indicating physical form are not symbolic in the way diagrams are, and not all drawings of 3D objects are necessarily meant to capture literal shape. For example, many consumer products

Table 4.2 Kinds of elements to be recognized.

\begin{tabular}{|c|c|c|}
\hline "What" to recognize & Examples & Remark \\
\hline Genre & $\begin{array}{l}\text { Mathematical graph, } \\
\text { architectural floor } \\
\text { plan, web site layout, } \\
\text { circuit design. }\end{array}$ & $\begin{array}{l}\text { It may be sufficient to recognize a } \\
\text { sketch is of a certain kind without } \\
\text { asking the user. The program } \\
\text { could assume the sketch is in a } \\
\text { certain domain. }\end{array}$ \\
\hline Characters (writing) & $\begin{array}{l}\text { Alphanumerics, math } \\
\text { symbols. }\end{array}$ & $\begin{array}{l}\text { Usually with other characters, in } \\
\text { words and sentences. }\end{array}$ \\
\hline Geometric shapes & $\begin{array}{l}\text { Dots, lines, rectangles, } \\
\text { blobs. }\end{array}$ & $\begin{array}{l}\text { Geometric shapes are often drawn in } \\
\text { relation to others. }\end{array}$ \\
\hline Spatial features & $\begin{array}{l}\mathrm{A} \text { is contained in, is } \\
\text { above, is larger than } \mathrm{B} \text {. }\end{array}$ & $\begin{array}{l}\text { Spatial relations among elements } \\
\text { may influence recognition. }\end{array}$ \\
\hline Entities & $\begin{array}{l}\text { Domain-specific notation } \\
\text { such as diodes, } \\
\text { transistors. }\end{array}$ & $\begin{array}{l}\text { Contextual clues can help } \\
\text { disambiguate semantics of domain } \\
\text { symbols. }\end{array}$ \\
\hline Artistic nuance & Shadows, textures, color. & $\begin{array}{l}\text { Ink that modifies an existing } \\
\text { element, perhaps suggesting } 3 \mathrm{D} \\
\text { shape. }\end{array}$ \\
\hline Commands & $\begin{array}{l}\text { Object selection, delete, } \\
\text { copy, move, etc. }\end{array}$ & $\begin{array}{l}\text { Command ink specifies operations on } \\
\text { the drawing. }\end{array}$ \\
\hline Intention & $\begin{array}{l}\text { Drawing's function or } \\
\text { behavior, e.g. circuit } \\
\text { breaker. }\end{array}$ & $\begin{array}{l}\text { Requires detailed domain knowledge } \\
\text { and reasoning. }\end{array}$ \\
\hline
\end{tabular}




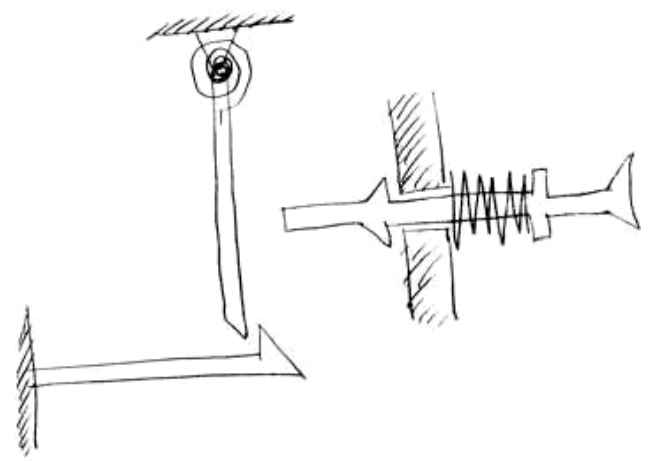

Fig. 4.1 A sketched circuit breaker [159].

come with assembly instructions featuring simplified diagrams of 3D objects to draw attention to only those features and relationships relevant for assembly.

Perhaps the most useful aspect of a sketch is the intention it captures. The circuit breaker shown in Figure 4.1 probably would not work correctly if it were manufactured exactly as shown, yet the intent of the drawing is clear to a person familiar with such engineering sketches [159].

\subsection{How Much Recognition is Appropriate}

The nuSketch Battlespace system shows that a sketch-based system need not support recognition at all [43]. It aims to enable users to quickly work with spatial data and issue commands that operate on that data. To support this goal, nSB only utilizes some properties of the user's ink. For example, the boundary or center of the ink may be important, but the system need not recognize the ink as a particular symbol.

Designers often create paper "storyboards" for showing high-level structure or expected behavior without needing to specify details. DENIM (for web site designers) and DEMAIS (for multimedia designers) allow users to create such storyboards $[16,99]$. Both these systems recognize a limited subset of the user's sketch input. Ink that is not interpreted as belonging to a special set of gestures or symbols is simply 
left on the canvas. This allows designers to capture ideas by sketching naturally without being interrupted by unwanted feedback.

\subsection{Segmentation and Grouping}

Segmentation and Grouping are related processes of breaking down user input and finding related ink. Segmentation involves partitioning undifferentiated sketch input into parts (analogous to identifying word boundaries in speech recognition [25]). Grouping is the process of forming logical collections of data (analogous to determining which spoken words compose a sentence).

It is sometimes appropriate to break apart continuous pen strokes into multiple segments, for example in recognizing cursive writing, or finding corners in continuous pen strokes. Alternately, it may be necessary to group related strokes in order to recognize compound objects such as a triangle drawn with three distinct strokes. To further complicate matters, there may be a number of reasonable ways to segment user input [104], based on information such as pen speed, stroke order, perceptual qualities, domain knowledge or curvature [84]. Multi-modal systems may be able to use additional information such as a user's speech or pointing gestures to help segment and recognize sketches $[8,126]$. Which segmentation technique is appropriate depends on what kind of sketch input the application expects. The technical challenge is simplified if the system requires users to complete each symbol before moving on to another, or if each symbol must be drawn using a conventional stroke order. However, such requirements work against the goal of supporting unconstrained, fluid sketching.

The following describes three strategies for segmenting and grouping continuous input strokes. They include the use of temporal data, perceptual organization, and delimiter-based approaches.

\subsubsection{Temporal Segmentation Techniques}

A common and effective method for segmenting ink is to use time. Temporal data comes in (at least) three flavors. First, we may look at the order strokes are made. For example, the letter $t$ is usually drawn with the vertical stroke followed by the horizontal crossing. Second, 
timing information for individual $(x, y)$ points tells us how fast the user was drawing at any given location, which helps identify corners. Last, a significant delay between individual strokes may be interpreted as a delimiter between elements, just as a pause in speech may indicate two distinct sentences.

Even for simple sketches there may be a very large number of possible ways to segment ink. We can use stroke ordering to reduce computational complexity of grouping and recognition [152]. Sezgin notes that common depictions (like stick figures) tend to be drawn using one of a small set of stroke orderings. However, it is also common for people to begin drawing one recognizable entity $X$, move to another, and return to complete $X$ later on. For example, many people cross their $t$ 's and dot their $i$ 's after writing all letters of a word. In Sezgin's terms, the user's marks are interspersed.

Another use of time for grouping ink looks at the velocity of the stylus as the user moves it, to identify locations of interest such as corners $[31,150]$, or places where the drawer may be taking extra care to be precise.

A series of strokes with short intervals may constitute a recognizable entity. For example, people may draw a compound entity like a television as a square enclosing a circle with a $V$-shaped antenna along the top. People tend to draw the three parts right after one another, and then pause to think about what to do next. A significant pause between pen activity might delimit objects. Such timeout-based approaches are easy to implement but some users find it imposes an unnatural feel to the application [40, 182].

\subsubsection{Perceptual Organization}

Spatial data can also help with ink grouping. A simple location-based approach forms groups based on ink proximity: if stroke $A$ overlaps or comes close to overlapping stroke $B$, group them together. However, this strategy breaks down if distinct entities overlap.

Gestalt psychologists offer the laws of perceptual organization to explain how people make sense of visual scenes. Humans perceive scenes not only using what is shown but also with "invisible extensions as 


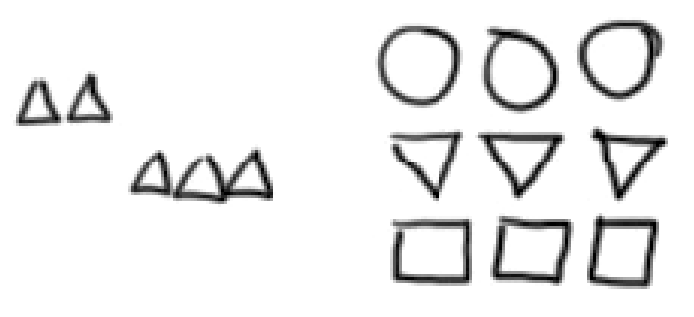

(a) (b)

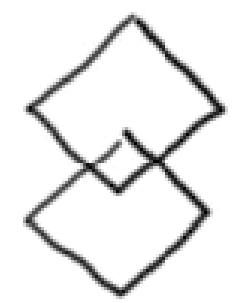

(c)

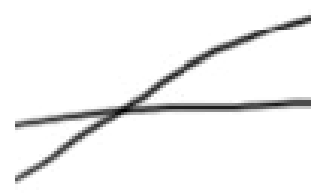

(d)

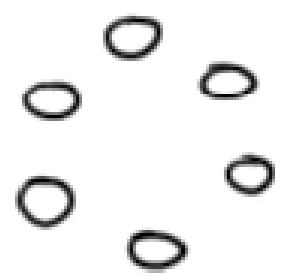

(e)

Fig. 4.2 Some principles of perceptual organization [78]. (a) Proximity: elements near one another are seen as belonging to a group. (b) Similarity: objects sharing features such as shape belong in the same group. (c) Symmetry: two shapes symmetric about horizontal and vertical axes, suggesting they belong together. (d) Continuation: the simplest explanation is two straight lines, not four lines meeting in the middle. (e) Closure: a large circle emerges from an arrangement of smaller circles.

genuine parts of the visible" [11]. The rules of perceptual organization include proximity, similarity, closure, continuation, and common fate [78] (see Figure 4.2).

Perceptual organization supports grouping at many levels. At a low-level, we can use perceptual rules to analyze the relationship among individual ink strokes to find plausible groupings for recognition. Mahoney and Fromherz show how perceptual organization rules can reduce the number of plausible stroke groupings into a ranked list of groups, which helps improve recognizer efficiency [103]. For example, an object may be drawn on top of background elements, as illustrated by the stick figure and horizon from Figure 2.2. Because the horizon has strong continuity from the left to the right of the stick figure, it is plausible the two halves should be grouped. The marks forming the stick figure are in close proximity, and share similar sizes, suggesting they may form a logical whole. 
PerSketch and ScanScribe explore how perceptual organization rules can be used on a number of levels [143, 144]. Drawings are analyzed in a manner approximating Marr's three-stage visual information processing theory [106]. In the early phase, ink is broken into "elemental curve fragments" called Prime objects (Primitive objects in ScanScribe). In the middle stage, Prime objects are put into plausible groups using perceptual organization rules. These groups are called Composite objects. Composite objects may include Primes as well as other Composites. In the last stage, domain rules are applied to combinations of Composite objects, identifying which combinations of composite objects are reasonable according to the subject matter (e.g. chemical modeling or electrical engineering).

Gennari et al. [48] propose a segmentation approach based on finding dense areas of ink and areas where the perceptual qualities of the ink changes. The Gennari system analyses input as the user draws, calculating features such as ink density. This approach is designed to work for node-and-edge diagrams whose symbols do not overlap.

\subsubsection{Context-Aware Segmentation}

Particular aspects of the domain's graphical language may afford the opportunity to use clever strategies for segmenting ink. This section describes two methods for forming groups of marks by analyzing ink.

Kara and Stahovich's SimuSketch is a sketch-based interface for creating graphical node-and-edge network diagrams in the Simulink application. Simulink supports users in simulating dynamic systems with a visual programming approach wherein boxes (nodes) represent functions or processes, and connectors (edges) represent inputs and outputs. SimuSketch looks for markers in the input sequence - easily identifiable symbols used to group other (nonmarker) ink. In particular, SimuSketch looks for edges between nodes (see Figure 4.3).

Shilman's work on parsing handwritten notes incorporates context awareness for discerning the structure of a sketched document [154] (see Figure 4.4). Shilman's algorithm integrates two related tasks in document analysis. The first challenge is to discern marks as either writing or pictures. The second challenge is handwriting layout analysis-grouping 


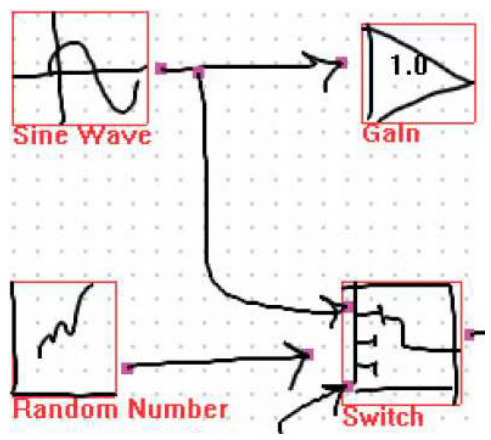

Fig. 4.3 SimuSketch exemplifies delimiter-based, multi-phase parsing of sketches. The first stage identifies arrows as delimiters. In the second stage, clusters of remaining ink are recognized as domain symbols.

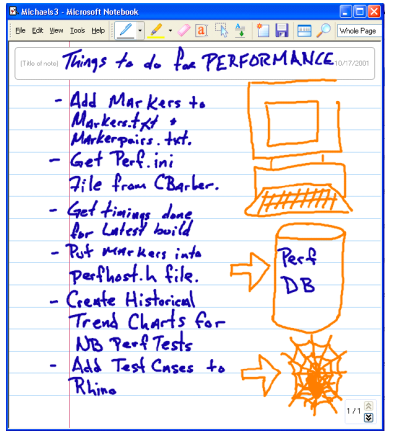

(a)

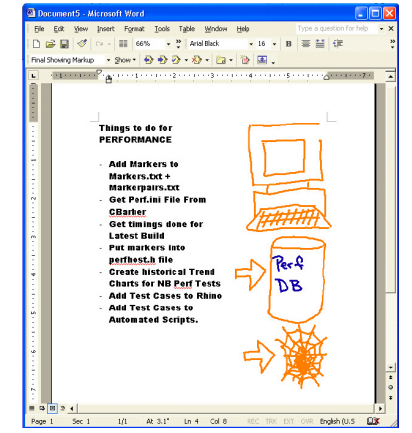

(b)

Fig. 4.4 Analysis of hand-written notes discern writing from drawings. Left: the original electronic document. Right: the same document after handwriting has been recognized [154].

ink that has been identified as writing into compound entities such as words, sentences, and paragraphs. By combining these two processes, Shilman et al. can perform a limited feature-based recognition on ink in order to test if it is text. The intuition is that "if you look at a page of ink with squinted eyes or from a distance, you can distinguish writing from drawing by its regular, linear structure." This approach uses features such as stroke length and curvature as well as information derived from the spatial and temporal relationship between fragments of ink. Marks are labeled as either "text" or "drawing" using these local and global features with a decision tree classifier. 


\subsection{Overview of Recognition Techniques}

This section gives a brief overview of various approaches for performing sketch recognition.

Regardless of the input device, we identify two broad categories of sketch recognition: online and off-line. Online recognition is analogous to your friend watching you sketch. Your friend may see how fast you make a stroke, in which order you make marks, and perceive what you say and how you gesture as you sketch. Interpretation happens as the drawing is made. Off-line recognition is analogous to your friend seeing your sketch for the first time after you have finished it. Recognition happens after the drawing is complete, irrespective of the order or speed strokes were made. While off-line recognizers only have access to the finished drawing, online recognizers potentially have access to data such as pen pressure, time, speech, and gesturing.

Pen input is generally captured as a sequence of time-ordered $2 \mathrm{D}$ coordinates. Other data such as pressure or (in a multi-user environment) user identity may be available depending on the input hardware. A single sequence of ink captured while the stylus is touching the drawing surface is commonly called a stroke.

Online recognition strategies are further divided into two categories: single-stroke and multi-stroke recognizers. Single-stroke recognizers are appropriate for tasks such as interpreting freehand gestures. Singlestroke approaches are simpler to implement than multi-stroke strategies because user input is clearly divided into pieces. This process could be relatively simple: a multi-stroke recognizer might simply expect multi-stroke objects to be drawn in a prescribed fashion. Or, multi-stroke recognizers might be more complex, for example hypothesizing which individual strokes (or segments of strokes) belong together, using Markov models or Bayesian Networks to aid hypothesis testing.

We need some way of representing the entities for recognition. By "entity" we refer to classes that may be recognized: boxes, lines, chairs, AND-gates, and so on (see also Table 4.2). The examples a recognition system has learned are called classes, and user's input are candidates. Three methods of presenting classes are covered here: hard-coded, 
visual example, and textual description. Particular instances of these methods are described later.

\subsubsection{Hard-Coded Recognizers}

One common approach is to hard-code recognition routines directly. For simple or limited graphical vocabularies this may be appropriate. A circle (or a zero, or the letter "O," or the sun, etc.) can be recognized with a short program looking for input points that are roughly equidistant from the stroke's centroid. However, ad-hoc, hard-coded recognizers are difficult to maintain and extend. For example, if we wish to extend our circle recognizer to interpret a sun with rays of light coming out of it, we would have to also recognize lines, then coordinate the recognizer to consider those particular lines together with the circle, and ensure that the lines are positioned and angled correctly. Further, sketch recognition applications must be able to discern different kinds of elements. The recognizers for each of these elements may conflict. A new recognizer may cause an existing recognizer to stop working correctly, leading to maintenance, debugging, and testing problems.

\subsubsection{Visual Matching}

Another strategy for representing classes is to create a library of drawn examples. Some techniques that use this approach are the Ledeen recognizer [123], the Rubine recognizer [138], Quill (based on Rubine) [102], Kara's recognizer [80], and the $\$ 1$ Recognizer [179]. The accuracy of some of these approaches can be improved if multiple training examples are provided. Other techniques require only a single training example.

Some of these approaches are feature-based. Such strategies compute properties such as stroke length, stroke path, minimum or maximum angle, or aspect ratio. A user's candidate entity is compared with classes using these features. An alternate approach is to treat visual examples as graphical templates, where candidates and classes are compared using some form of distance function.

User tests of recognition systems employing visual libraries commonly include 30 or fewer classes [80, p. 13]. For many applications a database of that size is appropriate. It is unclear how large these 
database can be: on one hand, human users have limited ability to recall how uncommon symbols must be drawn; on the other hand, recognition systems must search its (potentially extensive) library for matches. The larger the database, the greater probability of matching multiple classes, possibly leading to ambiguity.

Library-based recognition schemes differ in how quickly the algorithm searches its class database. Quill and the Rubine Recognizer compare user input to each symbol, while other strategies (such as Kara's recognizer) use efficient methods that exclude large portions of the library before performing computationally intensive comparisons on the remaining symbols. All of the methods discussed in the previous paragraph provide interactive performance on the target hardware. For example, the $1 \$$ recognizer produced results within about one second using a consumer-grade PDA from 2006 with 16 symbols in the recognition library [179].

\subsubsection{Textual Description}

Classes may be described textually using a programming language $[17$, $26,63,128]$. These notations have two primary strengths. First, humans can read them. The television symbol described in Section 4.4.1 may be described with natural language as "a square with a slightly smaller circle positioned at its center." A formal symbolic language for that statement is still quite legible (assuming one understands the function semantics):

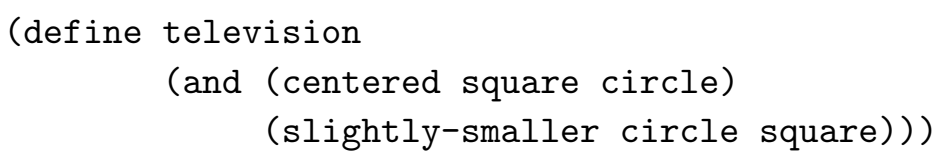

Another strength of this kind of notation is that it allows the developer to describe entities at a level of abstraction that accommodates variability between entity instances. An abstract triangle is a threesided, two-dimensional convex shape whose internal angles sum up to $180^{\circ}$. A particular triangle may have side lengths of 3,4 , and 5 , and be oriented so that its long edge is horizontal. We may define triangles and other entities as abstractly or concretely as the language allows. 


\subsubsection{Managing Ambiguity}

Futrelle's classification scheme of types of ambiguity in diagrams includes two high-level categories: lexical and structural ambiguity [45]. Lexical ambiguity refers to the "word" level, when the meaning of a particular symbol is in question. Structural ambiguity refers to confusion arising from the composition of symbols.

Shilman augments this scheme with two additional types of ambiguity that arise in sketch recognition: label and attribute ambiguity [153]. Label ambiguity is present when the symbol's identity is unclear. For example, a quickly drawn rectangle might be interpreted as a circle. Attribute ambiguity refers to the features of a sketched element: the exact location of a quickly drawn rectangle's corner may be unclear.

Two sketching systems that focus on managing ambiguity at different stages of user interaction are BURLAP and SketchREAD. A technique built into BURLAP [104] addresses domain independent ambiguity management at the GUI toolkit level, while SketchREAD [4] offers a method for using domain knowledge to disambiguate domain symbols.

BURLAP is a calligraphic application based on SILK [92] that enables people to draw user interfaces. As the user draws, BURLAP forms a list of plausible interpretations. At some point the system may need to pick one interpretation. Mankoff et al. call this process mediation, performed by agents called mediators. Some mediators may engage the user by displaying a pick-list of choices or visually indicating the ambiguity. Other mediators automatically execute and do not involve the user.

BURLAP exemplifies a framework for mediating ambiguity in recognition-based interfaces called OOPS [105]. OOPS consists of a library of error correction and ambiguity management techniques that work with existing GUI toolkits at the input-event level.

Alvarado and Davis's SketchREAD system demonstrates a technique for modeling recognition ambiguity [4]. The SketchREAD application is a domain-independent sketching tool. It accepts a textual description of classes to learn to recognize in a particular domain [63] 
(see Section 4.8). SketchREAD uses Bayesian Networks to reason about the user's input based on domain understanding [5].

Bayesian Networks also allow us to encode relations of compound objects that can further influence the belief in a hypothesis. Alvarado and Davis give an example from a circuit design application [4]. A diode is depicted as a triangle with a line tangent to one of the corners and parallel to the opposing triangle edge (see Figure 4.5). A person typically draws a triangle first. The system calculates the probability $P$ (triangle) that the marks depict a triangle. In circuit diagrams, triangles are strongly correlated with diodes, so at this point there is a partial hypothesis that the person is drawing a diode. Next, when the diode's line is drawn the system calculates $P($ line $)$. Because a diode consists of a triangle and a line, the Bayesian Network tests the hypothesis that the drawing is a diode by calculating the joint probability of the triangle and line interpretations. Even if one of the two parts (triangle or line) were drawn sloppily, the higher-level hypothesis diode can guide us to a meaningful interpretation. If additional information is present (such as connecting wires), additional belief values can be used to support or reject the hypothesis that the drawing depicts a diode.

A nice property of this recognition strategy is that it supports efficient re-interpretation on an ongoing basis as the user continues to work. The system endeavors to make sense of each new input stroke, making use of established interpretations and associated probabilities. While naïve systems may exhaustively test all possible interpretation hypotheses, SketchREAD's hypothesis pruning enables the system to test only the most likely interpretations. SketchREAD's interpretation speed scales roughly linearly with the number of strokes, compared to the exponential runtime used by some other approaches.

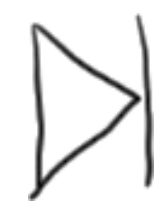

Fig. 4.5 A diode is drawn as a triangle with an adjacent line. 


\subsection{Pattern Recognizers}

Various methods have been investigated for performing recognition of sketch input. The methods covered have been known variously as character recognizers, glyph recognizers, and pattern recognizers. They operate on isolated strokes or segments of strokes and do not perform higher-level reasoning based on domain semantics or context.

All these recognition approaches work using the same general strategy, and although each has its own strengths and weaknesses, no single one is best for everything. The general strategy has not changed since the earliest online character recognition systems [56, 123]. The first step is to learn a dictionary of classes. These serve as instances of the elements the recognizer can handle. The recognition system converts user input into candidates. The candidate is then compared to classes in the dictionary, producing a similarity metric for each comparison. Sketch-based applications can use higher-level methods to reason about the most likely choice or choices. Alternately, the best match from the low-level recognizer might be used.

While the recognition strategies described below share a high-level strategy, they differ in how they represent candidates and classes and how they are compared.

\subsubsection{Ledeen Recognizer}

The Ledeen character recognizer is efficient and easily trained [123]. User input is scaled to fit inside a three-by-three grid of nine cells. Each stroke's starting cell is noted, and each stroke's path through the grid is encoded. This approach accommodates multiple stroke input but does not specify how multiple strokes should be grouped to be considered together. Newman and Sproull suggest considering multiple strokes together if less than half a second of latency separates strokes.

Some user input is not easily handled with this scheme. For example, straight vertical or horizontal lines would require scaling one dimension significantly more than another, leading to an unreliable path order. Dots also give this approach trouble. Problematic strokes like dots and vertical or horizontal lines are recognized by special-case algorithms instead of the $3 \times 3$ grid approach. GRAIL employed a 
multi-stroke, context-sensitive recognition strategy very similar to the Ledeen recognizer [56].

\subsubsection{Rubine Recognizer}

Rubine's feature-based recognition approach was demonstrated by a system called GRANDMA (Gesture Recognizers Automated in a Novel Direct Manipulation Architecture). The features involve geometric properties of single strokes such as start/end locations, total gesture length, sine/cosine of initial angle, and so on. Each feature must be calculable in constant time to ensure efficiency.

Some features are more effective than others for classifying a given symbol. If a number of examples are provided (typically 15 or more produce good results), the trainer uses linear discriminant analysis to determine the most effective features for classifying a particular entity.

Rubine's work was extended with a recognition system called gdt (later renamed Quill) [102], and added several feature types such as aspect ratio and curviness. Quill was incorporated into SATIN [69], a toolkit for building sketch-based applications.

\subsubsection{Kara's Recognizer}

A recognition technique developed by Kara and Stahovich [80] differs from stroke-oriented, feature-based approaches such as Rubine's. Instead, it compares the spatial distance between down-sampled bitmap representations of symbols. Kara's recognizer does not rely on geometric features like corners, angles or lines, and operates on input regardless of temporal information. Further, it accommodates multi-stroke entities as easily as single-stroke entities.

The technique first applies a polar coordinate transformation about a cleverly chosen pivot point. Input is incrementally rotated from $-\pi$ to $+\pi$ radians and compared with a class entity to determine a rotation that best aligns two symbols. This "pre-recognition" step helps exclude unlikely matches.

After rotating the user's input the algorithm creates an $n \times n$ downsampled bitmap ( $n=48$ works well). Next, the recognizer applies four spatial distance classifiers to compare user input against known classes. 
The results of each classifier are converted into comparable forms and combined to produce a single recognition value describing the similarity between the user input and classes.

\subsubsection{Cali Recognizer}

Fonseca et al.'s nontrainable Cali recognizer can identify geometric shapes at any angular orientation or aspect ratio [40, 41]. These shapes may consist of any number of strokes, as they are segmented based on a timeout (see Section 4.4.1). To identify the shape (triangle, cross, etc.) Cali first finds geometric properties based on the input: the convex hull, the largest triangle and quadrilateral inscribed inside the hull. Additional geometric properties are calculated based on these shapes, including areas and perimeter values and aspect ratios. These values are then used to search through prior statistical data regarding the shapes to be recognized. This approach uses fuzzy logic to determine membership in statistical equivalence classes. For example, the recognizer for the shape "Line" reports true if the input's aspect ratio "is very thin."

A trainable variant of Cali compared several learning algorithms for building the statistical equivalence classes: K-nearest neighbors, inductive decision trees, and Naive Bayesian Networks. Testing showed Naive Bayesian Networks the easiest to train with the best recognition rates.

\subsection{5 \$1 Recognizer}

The $\$ 1$ Recognizer [179] is notable because of its ease of use, algorithmic elegance and power. This technique is designed for use on PDA-like devices that accept pen-based, gestural input for characters and commands. The $\$ 1$ Recognizer requires only a single training example to be effective. Users may easily create their own gestures - it is trainable on the fly.

The algorithm processes candidate and class input the same way. It begins by resampling input into a number of points ( $n=64$ was found adequate). This enables $\$ 1$ to compare strokes of different sizes and drawing speeds. Next, the input is rotated so the angle formed by the input's centroid and initial point is $0^{\circ}$. After rotation the input is scaled 
nonuniformly to fit inside a square. Like the Ledeen algorithm, the $\$ 1$ Recognizer must handle certain classes of input (e.g. straight lines) differently because scaling the input nonuniformly would distort it too much. Last, the candidate is compared to each class in the dictionary by summing the error of corresponding points.

\subsubsection{Graph Matching Techniques}

The techniques discussed earlier are best used for online recognition because they require timing data regarding stroke path (e.g. Ledeen's approach) or where strokes begin and end (e.g. $\$ 1$ or Kara recognizers). But online recognition is not always possible. A designer may scan a paper sketch and expect to use it as the basis for further work. In such cases, off-line recognition might be employed. Graph-based recognition techniques are commonly used for this purpose in the document analysis and engineering diagram research community.

The first step in graph-based off-line recognition is to convert the input image from a raster to a vector representation [166]. Vector endpoints and junctions form graph nodes, and edges represent lines between them. Because graph nodes contain additional information such as $(x, y)$ position, these structures are called attributed relational graphs.

A full drawing's corresponding graph can be searched for identifiable portions, or subgraphs using subgraph isomorphism algorithms [97]. Lladós has proposed an heuristic technique for error-tolerant pattern matching using subgraph isomorphism [101]. For example, suppose we are interested in finding the pattern shown in Figure 4.6(a) in the test image shown in Figure 4.6(b). After converting both the example pattern and the test image to attributed relational graphs, this technique begins searching through the test image graph for sequences that resemble the example pattern's graph. When this algorithm finds a near match, it employs edit operations on the test image graph to force an exact match. Recognition confidence is measured in terms of how much the observed graph must be edited in order to match the expected graph. As shown in Figure 4.6(c), the approach is rotation and scale invariant and tolerates some degree of distortion. 


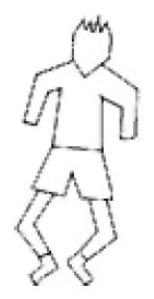

(a)

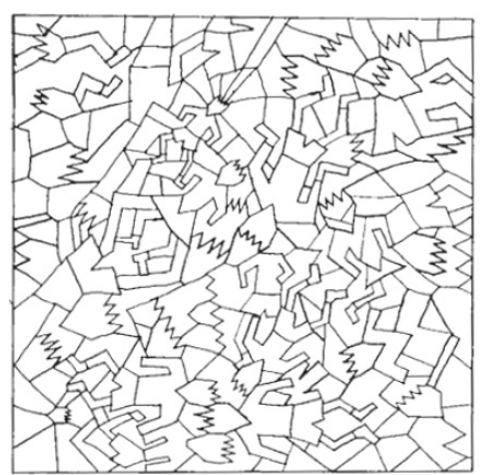

(b)

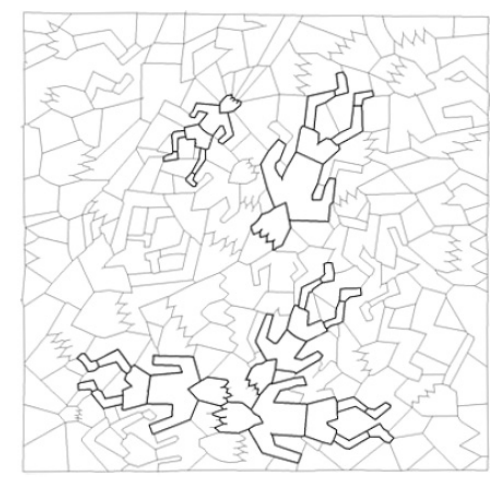

(c)

Fig. 4.6 Example of graph-based pattern matching [101]. An example pattern is shown in (a). The complex test image in (b) contains several distorted, scaled and rotated instances of the pattern. Portions of the example pattern found in the test image within acceptable error tolerance are shown in (c) with thick lines.

\subsection{Recognition of 3D Scenes}

Although many sketches represent abstract entities that lack physical form (e.g. UML diagrams), many others have physical, 3D interpretations. In this section, "recognition" refers to reconstructing 2D sketch input as 3D models. In this sense the recognition is syntactic (e.g. identifying a rectangular parallelepiped) rather than semantic (e.g. identifying a shoe box). Reconstruction of 3D scenes has been studied in the robotics and computer vision community as well as by CAD researchers.

Three-dimensional recognition is essentially "reverse projection." The strokes on the drawing surface provide $(x, y)$ data but not depth (z) [100]. Because $z$ could take on any value there are an infinite number of ways the $2 \mathrm{D}$ drawing could be turned into a 3D model. Fortunately knowledge of solid geometry restricts the possible interpretations: the surface of a 3D object cannot pass through itself, so any interpretation that involves self-intersecting surface planes is invalid. There are many ways to compute particular $z$ values for sketched 3D models. Analytic approaches include line and junction coding [23, 71] and solving linear equations [55]. Other approaches attempt to fit sketch input to likely constructions such as primitive 3D structures such as 
cylinders [175], or by finding likely geometric constraints like parallelism and symmetry [155]. Many 3D sketch recognition systems employ a combination of these approaches.

People can recognize sketches as long as they are made from a familiar vocabulary. We also make inferences about $3 \mathrm{D}$ shape based on $2 \mathrm{D}$ perceptual qualities such as parallelism, right angles, and alignment to primary axes. Further, drawings of 3D objects often use shading to show shadows and contours. Frequently when people draw a 3D object, they draw only the visible contours, leaving the rest to the imagination. It is not difficult to look at a box and be able to "see" the edges and corners that are obscured by the solid geometry (although the imagined edges might in fact be wrong). Some have developed heuristic methods for hidden-line inference $[82,115]$ that "reconstruct" a 3D object without access to a complete 2D wireframe. A wireframe model shows edges in a "see-through" view; hidden lines are shown through surfaces.

Design software need not reconstruct full 3D models to be useful. Instead, the recognition system could build a $2.5 \mathrm{D}$ interpretation of a $2 \mathrm{D}$ sketch. A $2.5 \mathrm{D}$ model contains depth information only for geometry visible from a single vantage point, so the recognition system is relieved of the difficult task of inferring the full 3D structure. Recent work from Microsoft Research Asia demonstrates an interactive 2.5D recognition and rendering system [22]. Figure 4.7 shows sample output of this approach.

\subsubsection{D Curve Analysis}

Three-dimensional recognition begins much the same way as $2 \mathrm{D}$ recognition. Individual marks constituting a drawing are classified into

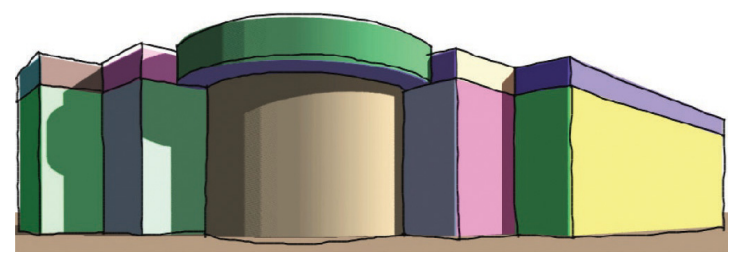

Fig. 4.7 "Sketching reality" allows users to create 2.5D architectural models by sketching [22]. 
features such as lines, curves, and junctions. The recognizer must determine the relationship between these features. For example, if a line represents an edge between two faces, what is the dihedral angle? There are a number of ways to perform this analysis. Two are discussed briefly below.

One approach to 3D stroke analysis is to label drawn and emergent elements such as lines, vertices, and faces. The Huffman-Clowes method [23, 71] identifies various ways' faces of a drawn polyhedron may relate (see Figure 4.8). This helps reconstruct the 3D geometry of the object based on the 2D drawing. A Huffman-Clowes label indicates whether the line represents a concave or convex edge. Edges are labeled "obscuring" if one of the adjoining faces is not visible. Given a line drawing, there may be more than one possible way to assign Huffman-Clowes labels [165] (see Figure 4.9). Schweikardt and Gross used Huffman-Clowes to interpret sketches of 3D objects in their Digital Clay system [149].

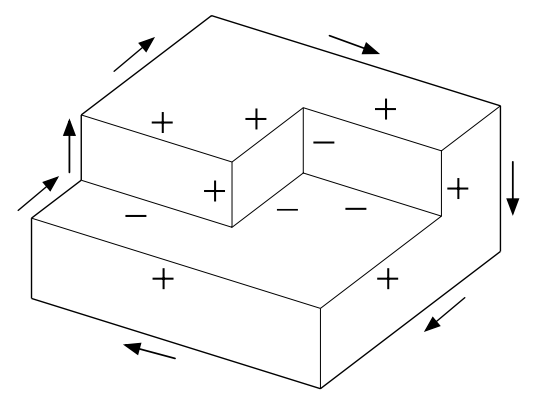

Fig. 4.8 An illustration of Huffman-Clowes labels. Arrows indicate edges whose faces are obscured. + and - identify edges as convex and concave.

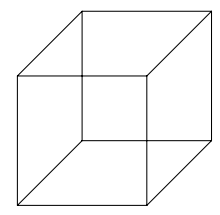

(a)

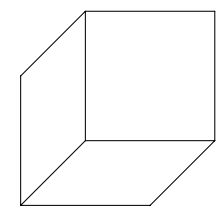

(b)

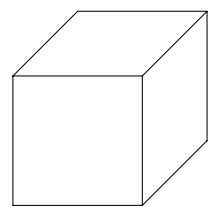

(c)

Fig. 4.9 A Necker Cube illustrates difficulty when forming 3D interpretations of 2D line drawings. The wireframe shown in (a) can be interpreted in two equally valid ways, shown in (b) and (c) with hidden edges removed. 
Shpitalni and Lipson use regression to classify input strokes and clustering to identify 3D vertices shared by multiple lines. Stroke classification fits input to conic sections using a linear system of equations [155]. This approach is effective for detecting straight lines, parabolic arcs, and hyperbolae. A sharp (or filleted) corner, for example, can be found with a hyperbolic conic section. After detecting curves, neighboring curve endpoints are merged when appropriate.

\subsubsection{Gesture-Based 3D Recognition}

One way to provide input for a 3D sketching system is gestural commands for creating or editing solid geometry models. SKETCH [182] and SKETCH-N-MAKE [19] recognize gestural commands to represent certain 3D forms. For example, when the user draws two parallel lines, the system generates a cylinder. The drawing direction defines whether the solid is additive (upward gestures) or subtractive (downward gestures).

\subsubsection{Direct and Indirect Solid Model Drawing}

Teddy [75] interprets user input as the outline of an object. The object is then inflated to provide a smooth, blobby object. Subsequent edits are done by explicitly changing modes and entering commands as appropriate. For example, an object in Teddy may be extended by drawing an attachment point on the existing object followed by an outline of the new part of the object.

Not all the user's marks denote object boundaries. Designers draw shadows, stipple dots, or little lines to indicate curvature or surface texture. Cohen leverages this to create 3D models from sketches by drawing shadows of objects [24]. In this system, ink is interpreted either as solid model geometry or as the shadow of that geometry projected onto a floor plane below.

\subsubsection{Imposing and Inferring 3D Axes}

Finding depth values for vertices in a 3D sketch is easier if the drawing conforms to an axis system, and if the object's faces are known to be generally parallel to these axes. It may be reasonable to impose a $3 \mathrm{D}$ 
axis scheme on the user. Alternately, the system can interpret a user's drawing and infer an axis system.

Chateau allows people to design 3D objects (like French villa-style buildings) using a constrained vocabulary of lines and angles [72]. In Chateau, ink is interpreted as wireframe elements that may connect to existing pieces on an explicit "drawing plane."

A coordinate system helps orient the user's marks so the overall geometry can be found. However, some 3D sketching systems do not provide a default 3D axis. The user may draw objects from any perspective. One approach forms an Angular Distribution Graph (ADG) showing how often lines are drawn at each angle [100, 108]. The ADG allows the software to infer a coordinate system based on an analysis of the drawing itself.

Tsang presents two methods for guiding 3D reconstruction [167]. In their system, recognition works in the context of two kinds of guides. First, a 2D image may be imported and placed in the drawing environment. The position and curvature of sketched input is influenced by these images. Second, the system analyses the drawing and suggests $3 \mathrm{D}$ wireframe models from a database composed of prior work by the user or by third parties.

\subsubsection{Styling Based on Existing 3D Geometry}

In many 3D sketching systems, the visual representation is often beautified during reconstruction from $2 \mathrm{D}$ to $3 \mathrm{D}$. Beautification may substantially change the character of the drawing so it no longer looks "sketchy."

The work by Nealen and colleagues builds on the Teddy-like approach of generating smooth $3 \mathrm{D}$ objects based on a sketch $[118,119]$. Users may draw on the surface of 3D objects as a way to issue modeling commands. To stretch a region of interest, the user first draws on the object to create the control points, then drags them to stretch the surface.

Often designers create new instances of known object types. For example, when designing the shape of computer speakers, designers work from experience with other speakers. By providing a 3D wireframe 
that is approximately the same shape as the desired object, a designer can sketch around the wireframe to create a new shape [79, 111]. The user's marks are recognized in the context of the wireframe, enabling the designer to quickly create a new styled instance of an existing class of objects without having to first make the wireframe.

A related approach is shown in the domain of organic plant modeling [6]. Instead of a geometric wireframe, this approach applies a mathematical model specifying the target domain based on knowledge of plant biology. This technique allows designers to quickly converge on a specific, highly refined 3D model by sketching. To be sure, this advantage comes at the cost of generality.

\subsection{Recognition Training and Domain Modeling}

In order for a sketch recognizer to interpret user input it first needs a representation of whatever elements it is intended to identify. There are two broad categories of methods for acquiring the model from users. The first is to provide drawn examples; the second is to provide written descriptions.

Many of the pattern recognizers discussed here provide user interfaces for capturing training examples. In general these all work the same way: users provide pen input and then label that input. The Rubine recognizer and Quill require multiple training examples to determine which analyzed features should be used to classify candidate input.

An alternative approach to providing drawn examples is to describe symbols with some sort of text description [107]. Costagliola et al. refer to these as Sketch Grammars, or SkGs for short [27]. An SkG is a BNF-like description of how low-level elements like lines and circles can combine to form higher-level elements. The combinations are described in terms of geometric shapes and constraints describing their relative lengths, positions, and angles. Once an entity's grammar is defined it may be used to compose more complex elements. The grammar-based recognition approaches covered here all share similar constraint types such as parallel, perpendicular, above, below, smaller-than, and centered-inside. 
Current work on sketch parsing leverages prior work from the visual languages community. Lakin describes visual language parsing as "the process of recovering the underlying syntactic structure of a visual communication object from its spatial arrangement." Lakin's vmacs system [90] supports users in providing unstructured graphic input that can optionally be parsed by a visual grammar in order to formally describe the structure of the depiction.

Grammar-based diagram parsing by Futrelle and Nikolakis focused on parsing scientific figures as they appear in publications [46]. This research used vector graphics rather than rough sketches, but it could be applied to interpreting hand-drawn diagrams.

Compound objects were defined in the Electronic Cocktail Napkin (ECN) as a set of elements and spatial relations [60]. The ECN generated symbolic constraint descriptions from the user's sketch input. The user could then modify the description by adding or deleting constraints, or generalizing or making them more specific. The ECN used a hybrid recognition approach involving a low-level pattern-based recognizer to identify symbols and a high-level grammar-based approach to recognize pattern configurations.

Constraints are often used to prescribe relations between elements during design ("ensure line A remains perpendicular to line B, even if line B moves"). But constraints may also be used to describe relations exhibited by recognizable elements. Rather than using constraints as rules for enforcing some conditions, they can be used to search for configurations that match known elements. Pasternak's ADIK system searches constraint declarations as a method for performing diagram recognition [128]. To use the above example, a user may define a plus symbol using constraints such as "line A is perpendicular to line B." Other constraints would specify the relative size and positions. ADIK constraints also specify tolerances so hand-made drawings could be recognized.

Hammond's LADDER language [63] builds on work pioneered by systems like ADIK by enabling users to textually describe how domain elements are drawn by stating the element composition and constraints governing those elements. LADDER allows programmers to prescribe how the element should be recognized, displayed, and how users could 
interact with the elements once recognized. For example, an object's "editing" definition may specify whether an object may be rotated.

Recognizers like Ledeen or Rubine learn based on drawn examples. A challenge with those approaches is that they must determine which features of the drawing are important and which are not, generally without user assistance. The salient features are extracted from training examples. Grammar-based approaches may be difficult to write, especially if recognizable classes have visual features that are cumbersome to describe verbally. After all, drawings (sometimes) encode nuances that are difficult to describe in words.

A third approach builds on the two others described above by combining the benefits of grammatical descriptions with drawn examples. Shilman describes a system similar to LADDER that incorporates the use of a statistical model for parameterizing the spatial relations between drawn elements [153]. Typically, a relationship such as "A is directly above B" is interpreted as either true or false based on some threshold values that give definition for the word directly. Shilman's approach allows the system to learn statistical distributions based on training set examples. In this case, the statement "A is directly above B" can take on various levels of truth. Shilman uses a labeling scheme based on a naïve Bayesian classifier.

Another hybrid approach involves generating grammars based on analysis of sketches [64, 172]. Users provide either a sketch or a textual description of a new element to recognize. The system then generates a shape that is close to (a "near miss") the user's original input. The user then adds or removes constraints as necessary to improve the match. This iterative process of shape generation and constraint refinement continues until the system produces a satisfactory model from the description of constraints.

This section has discussed several aspects of sketch recognition, including when, what, and how much to recognize. Algorithms for segmentation and reasoning have also been reviewed. Last, we described approaches for training recognition systems to learn symbols, make use of contexts, and understand domain semantics. The challenges in this section are primarily technical. The following section continues the topic of sketch recognition from the perspective of human interaction. 


\section{Interaction in Sketch-Based Software}

The computational support for sketching discussed in previous sections dealt with a wide range of topics, from hardware to data structures and algorithms. While previous sections focused on the technology itself, here we look more closely at people's interaction with sketches as mediated by computational systems.

Traditional design software uses a familiar array of interaction controls and idioms: buttons, toolbars and palettes, menus, double-clicking and right-clicking mouse buttons, scrollbars, keyboard shortcuts, and so on. With few exceptions, these approaches give the user unambiguous methods to express their intentions.

However, sketching design tools must "interpret" user input as messages that could have multiple meanings. If the user encircles an object, the system must decide if this constitutes selection, or if the circle is part of the existing object, or if the circle is part of some other object, or if the new ink is an annotation. The semantics of sketched user input may depend heavily on the domain as well as the functionality the application supports.

There are several high-level classes of operations the system might need to interpret: model, environment, and recognition/rectification operations. 
Model operations are those marks that specify creation of new, or modify existing, portions of the model. Some actions (such erasure) require a target or operand, suggesting the need for sketch-based selection techniques. Freehand annotations are important for informal work: they allow designers to record provisional ideas in context. Such notes might be considered part of the model. If the hardware supports it, pen properties such as pressure or angle could be used in subtle ways to signal different kinds of input — soft marks could indicate texture or shading, for example.

Environment operations are messages that change the state of the design tool itself in some way. They may specify changes in viewing parameters such as zooming, rotating, panning, or switching between "rough" and "rectified" renderings. Users might issue commands for performing some task immediately, from simple requests like printing to more involved tasks such as invoking a simulation. Environmental commands are often issued using buttons or menus, or they may be issued by gestures. However, interface components designed for keyboard and mouse hardware may be inappropriate for use in sketch-based systems.

Recognition operations allow users to initiate or interact with a process. Some systems enable users train the recognizer by specifying new objects or relationships among objects, which requires training techniques. The sketching system may also let users to trigger the recognizer rather than recognizing automatically. Some recognition systems are noninteractive and simply use the "best" interpretation. Other systems are interactive, enabling the user to choose among alternatives. As with some model operations, users may be allowed to select specific portions of their drawing for recognition.

The distinction between the above operation classes is often blurred. For example, a gesture to erase an object (a model operation) must be recognized before it is executed.

\subsection{Managing Recognition Error}

In any but the most trivial of tasks, sketch recognition will occasionally fail. Either the recognizer does not correctly interpret the user's sketch, or the recognizer is unable to produce an interpretation. Perhaps the 
user's sketch was drawn in such a way that even a human observer could not correctly interpret it. Maybe the user drew something the recognition system was not intended to handle. Regardless of the reason for the failure, it is important to manage problems that arise due to failed recognition. The particular strategy for managing error depends in part on when the system employs recognition.

Consider an application whose recognition process assigns a numeric confidence value describing how well a candidate entity matches entries in the recognition library. The multiple matches are put into an $n$ best list ordered by confidence levels. When the recognizer is obliged to report the most likely interpretation, it can simply respond with the item with the highest confidence value. Alternately the recognizer could respond with multiple interpretations [4, 60], but most applications are not designed to accommodate that. Recognition results may be inappropriate or inaccurate in a number of ways, summarized in Table 5.1.

The system may attend to inappropriate or inaccurate recognition automatically or interactively. Interactive methods include suggestive interfaces that provide alternative interpretations [72]. Some systems like BURLAP [104] use both automatic as well as interactive methods.

\subsection{Reacting to Sketch Input}

The system must determine when and how to react once it recognizes sketched elements. One action is to give the user feedback of recognition results. Textual, visual, and audio feedback have been used. Another type of action triggered by recognition is to invoke a command or program. Finally, recognized ink may be transformed into an interactive

Table 5.1 Categories of sketch recognition difficulties.

\begin{tabular}{|c|c|}
\hline $\mathrm{acor}$ & ser drew $X$ but the $\mathrm{r}$ \\
\hline Amb & $\begin{array}{l}\text { unable to confidently } \\
\text { ff } X, Y \text {, or } Z \text {. }\end{array}$ \\
\hline Uncertain: & $\begin{array}{l}\text { The user drew } X \text {, but the recognizer could not confidently } \\
\text { find any interpretation. }\end{array}$ \\
\hline Unintended: & $\begin{array}{l}\text { The system correctly recognized } X \text { but the user was not ready } \\
\text { to work with recognized elements yet. }\end{array}$ \\
\hline
\end{tabular}


object whose behavior is consistent with whatever it depicts, such as a sketched scrollbar turning into a manipulable scrollbar [92].

Taking action based on recognition events can be problematic because of the inherent ambiguity and uncertainty of sketches. Passive feedback of recognition (such as an n-best list menu) can be ignored. However, some forms of feedback may have lasting effects: a sketched gesture inaccurately recognized as a delete operation cannot be easily ignored.

The Electronic Cocktail Napkin could be configured to rectify (and unrectify) user input but it is turned off by default $[58,59]$. Rectification (sometimes called beautification) represents the user's input in a less sketchy form, and is one method of providing visual feedback. Some researchers feel that rectification is harmful because the input's rough character reminds viewers the design is unfinished. Alvarado's informal user studies of ASSIST suggests that the system should clean up a drawing after the user finishes rather than rectifying as they draw [2, p. 91].

Beautification is not always antagonistic to design, however. Published diagrams are usually "cleaned up" such that lines are made rectilinear, or other graphic elements are made the same size or similar spacing. Pavlidis and Van Wyk describe a system for beautifying rough drawings after they have been drawn [130]. This approach calls for automatic inference and satisfaction of graphic constraints.

While some systems wait for users to explicitly request rectification, Teddy renders user input as 3D shapes as they are provided [75]. Arvo and Novins demonstrate a novel method of rectifying 2D user input while maintaining the rough, hand-drawn qualities in the context of interactive sketches [13]. The lines have a "shape memory" that gives them a tendency to maintain their initial visual characteristics as users stretch, move, and bend them.

Do investigated the context and intention of hand drawn design diagrams in order to invoke appropriate tools [35, 36]. The Right Tool Right Time system is based on the observation that as people sketch, the symbols they draw might indicate the tools they need to support their activity. For example, an architect who is thinking about what is visible from a vantage point may draw sight lines on a floor plan. If the 
sketching program recognizes the user's input as sight lines, it could invoke a visual analysis program to show what can be seen from the indicated point.

\subsection{Toolkits for Sketch Recognition Systems}

Modern toolkits support programming of standard WIMP applications with a standard set of widgets such as buttons, drop-down lists, and scrollbars. These widgets are designed for use with a keyboard and mouse, using conventional interaction idioms like double clicking and drag-and-drop. Evidence suggests that these interface widgets and techniques are not always appropriate for sketch-based applications $[3,34,142]$.

Microsoft's Tablet PC API supports use of a stylus in pen-aware applications. The Tablet PC API excels at measuring and rendering user input. It also provides methods to access various features of freehand input such as sampling point locations, velocity, and pressure. Plimmer and Freeman have developed InkKit, a toolkit for building sketch recognition-based interfaces using the Tablet PC API [133].

SATIN [69] incorporates many engineering aspects of creating sketch-based applications including support for pen-centric widgets like pie menus (Figure 5.1) or pluggable recognizers (like Quill/gdt [102]). SATIN served as the basis for applications like DENIM [99] and SketchySPICE. However, no further applications have been built using this toolkit. A discussion of recognizers from various points of view (users, designers, and programmers) is presented in [70].

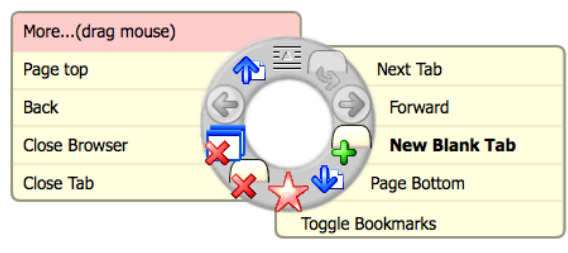

(a) Pie menu extension to the Firefox web browser.

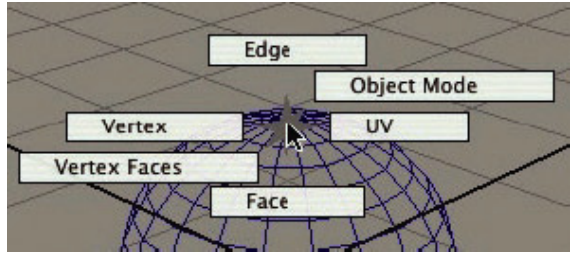

(b) Autodesk Maya pie menu.

Fig. 5.1 Pie menu implementations in two applications. 
The CrossY system features pen-centric user interface components such as those shown in Figure 5.2 [9]. High-level interaction toolkits for sketching incorporate not only widgets, but also support for ambiguity management and alternative recognizers.

BURLAP's mediation strategy (see Section 4.5.4) is part of the OOPS toolkit for managing ambiguity [105].

The Electronic Cocktail Napkin [59] served as a platform for developing other tools such as Right Tool Right Time [36], Stretch-ASketch [57], a Local Area Network designer [86], and others [60].

\subsection{Sketches and Human-Human Interaction}

People use sketches to interact with other people. When we draw a map to help somebody navigate to a location, the marks we make provide just enough information to communicate the route [169]. Such maps are media for human-human communication, and they serve their purpose well. When people make drawings to communicate everyday, mundane information, they tend to use consistent notation. Even when drawing to illustrate abstract concepts (such as "How a web search engine works") people use a limited visual vocabulary [66].

People interact with their sketches. This goes beyond the physical act of drawing. When we sketch, we make marks on the page, look at them, and potentially see new meanings in our drawing [52, 147]. We interact with sketches differently depending on many factors: size of drawing surface, kind of pen, relevant domain, expected sketch life cycle, how public or private it is, whether we are working alone or collaborating, and so on.

When collaborators work on problems together, they often use shared drawing surfaces like whiteboards or big sheets of paper. Mynatt et al. [117] looked at how people use whiteboards in practice and developed the Flatland system to support those activities. Marks on whiteboards are informal, and depending on their location they may be public. Whiteboards are used to record lists of ideas, items to buy, things to do, and so on. They also serve as an informal record of what is going on in the workplace. A colleague's unerased drawing may spark conversation among others long after the sketch's original purpose has been served. 
Computational whiteboard systems like Flatland, Colab [160], and Tivoli [113, 131] leverage the informal, quick, semi-public properties of traditional whiteboarding practice. At the same time, the marks on the electronic whiteboard become interactive: lists can be sorted, items checked off; text and drawings may be grouped, moved, scaled, and rotated. Physical whiteboards are usable only by those present; computational whiteboards may be used by distributed teams. This functionality has been demonstrated both by commercial collaborative conferencing tools and research prototypes such as the Workspace Navigator [77].

\subsection{Pen Interaction Techniques for Sketch-Based Systems}

An important consideration for developing new technology is the cognitive load a tool imposes [125]. Oviatt's study of high school mathematics students suggests that using a pen-based application on a tablet computer is significantly worse than working problems using traditional pencil and paper. Using the tablet, students took longer to complete math problems, and they did not like using the technology. Anthony et al. conducted a similar study comparing how students write equations using different input paradigms. The input methods included a standard WIMP interface and freehand writing performed with a computer stylus [8]. They found that students preferred pen input, and hand-wrote equations faster and more accurately than using the keyboard and mouse dialog. Paper and pencil was preferred to electronic writing, but pens of either sort are preferred over keyboard and mouse input. Both studies support the observation that the more attention users must allocate to using the tool, the less attention they can give to the problem at hand.

More natural interaction techniques must be developed in order to reduce cognitive load. Several methods have been shown to work well for pen applications. Marking menus are a good example [87]. Traditional menus provide a list of options, growing downwards and to the right. For pen devices with co-located input and output (such as a Tablet PC) the user's hand may obscure the menu. Pie menus (a type of marking menu) appear centered at the pointer location, with 
options distributed radially (see Figure 5.1). The user's hand may still be partially in the way, but at least part of the menu remains visible. The benefit of the marking menu is that people can learn to perform gestures without reading the option labels if the options appear in consistent locations. The need for a visual menu eventually disappears, leaving only the gesture. Pie menus are used in applications such as Maya [14], games such as the Sims [38], and are available as add-ons to web browsers. This approach works well, but has two drawbacks. First, it is not clear how the menu should be invoked. Second, gestures must be discovered, learned, and remembered.

Ramos et al. [136] further explored the use of pressure data for penbased interaction. In addition to the stylus $(x, y)$ position, pressure serves as another dimension the user can freely manipulate. For example, pressing lightly may produce a menu with one set of options, while pressing hard offers a different option set. Pressure could be effective as an input modality if used appropriately, including haptic and visual feedback.

Traditional menus are typically invoked by clicking the mouse, applying force orthogonal to the mouse's plane of operation, which does not cause the mouse to move. Some computer styluses have barrel buttons that allow users to "right click," but the force necessary to depress the stylus button is liable to cause unintentional pen movement, leading to mistakes [67].

Hinckley et al. [68] suggest the use of delimiters to trigger marking menus. A delimiter is "something different" in the pen input that the user is otherwise unlikely to draw but is easy to remember. One example delimiter in their Scriboli system is a pigtail mark, a loop made at the end of a selection gesture. This fluid motion lets the user indicate target objects followed by a command to apply to those objects.

Others have developed interface idioms for supporting pen and sketch input. Gedrics are gesture-driven icons for pen-based applications [47]. Each Gedric is associated with a class of tasks such as "changing font properties" in a text editor. To invoke a command, the user draws a gesture on a Gedric. The system recognizes the gesture, giving the icon meaning that can be activated by tapping it. On the "font" Gedric, drawing a slanted line might italicize selected text; drawing a 
vertical gesture from the bottom to top increases text size. However, while Gedrics help make some operations more convenient and reduce clutter on user interfaces, they also require users to know how to turn their intentions into a gesture.

While pointing and clicking is appropriate for tasks commonly performed with mice, Accot and Zhai suggest "crossing" as an apt technique for stylus interaction [1]. The experimental drawing program CrossY [9] demonstrates interface widgets that support crossing. To activate a CrossY button, the user draws a line from one side of the button to the other. Crossing makes it easy to take multiple actions with a single fluid motion, for example changing pen color and thickness by dragging the pen across adjacent Cross $\mathrm{Y}$ widgets (see Figure 5.2).

The inherent ambiguity of sketching is also present in pen-based interaction that is not intended to be sketchy, e.g. pressing a button or choosing a menu item. When the user attempts to act on an object the system may have to disambiguate the target. For example, when pressing a button, the pen may accidentally slide off one into another. This is called target ambiguity [104, 105].

An alternative to mediating ambiguity is to preempt it. Pegasus and Chateau $[72,74]$ demonstrate a suggestive interface that predicts what the user will draw and shows the outcomes of various possible actions. If a pick-list mediator from BURLAP/OOPS is like asking you to clarify what you have said, a suggestive interface is analogous

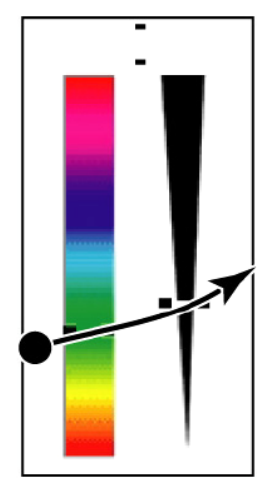

Fig. 5.2 Cross $\mathrm{Y}$ interface widgets let users change multiple parameters with a single stroke - pen color and thickness in this example. 
to completing your sentence before you finish. For example, Chateau's simplified domain of architectural design [72] is constrained to certain configurations of walls and beams known to produce good results. This technique is appropriate when the domain has a highly regular grammar or when structural properties such as symmetry may be exploited. Tsang and colleagues use a suggestive interface for their 3D sketching system that can model shapes such as aircraft hulls [167]. The system provides an overlay that guides users in providing additional sketch input. Users found this conducive to providing precise input. The system uses the incomplete sketch as a database query, finding similar drawings, and suggests additional geometry that may be appropriate.

Bae's 3D curve modeling system demonstrates how several calligraphic interaction techniques can be used together to provide a highly fluid sketching environment [15]. The system, called ILoveSketch, presents a physical sketchbook metaphor. Note that like Sketchpad, input is provided using a stylus, optionally modified by pressing physical buttons with the nondominant hand (Figure 5.3). The interface lacks the familiar on-screen buttons, scroll bars, and menus. Instead, users give commands with gestures which are often contextsensitive. For example, to browse earlier drawings the user "peels" back virtual pages by dragging a corner; to erase an item the user draws a "scratch-out" gesture. Many techniques implemented in ILoveSketch

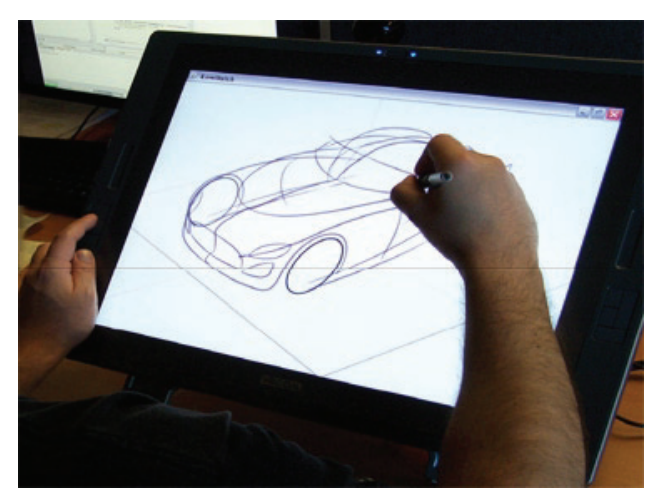

Fig. 5.3 ILoveSketch presents an "as natural as possible" sketching system that brings together many calligraphic interaction techniques [15]. 
address challenges associated with sketching 3D objects. For example, the system infers appropriate viewing angles by rotating or panning based on the user's work.

Alvarado provides a list of seven design guidelines for developing Sketch Recognition User Interfaces, or SkRUIs [3]. These guidelines are:

(1) Display recognition results only when the user is done sketching.

(2) Provide obvious indications to distinguish free sketching from recognition.

(3) Restrict recognition to a single domain until automatic domain detection becomes feasible.

(4) Incorporate pen-based editing.

(5) Sketching and editing should use distinct pen motions.

(6) SkRUIs require large buttons.

(7) The pen must always respond in real time.

While WIMP interfaces have been widely used since the mid1980s, sketch-based interfaces remain to be adopted. To develop better interaction guidelines, interface design patterns and toolkits, the research community must continue to build and evaluate sketchingcentric applications.

\subsection{The "mode problem"}

User interfaces often interpret input differently depending on which mode a program is in. For example, a structured graphics program may have input modes such as select, draw line, or fill color. Such a tool allows users to indicate rectangular areas when the selection tool is active. The same program also allows users to draw when the pencil tool is active. In both cases the user presses a mouse button and drags the cursor. But the program interprets user input in terms of the active tool. Sometimes users are unaware of which mode the program is in, or are unsure how to change to the desired mode. Managing modes often introduces cognitive load by forcing users to think about the tool rather than their work. This is called "the mode problem" [164]. It has been 
a challenge since the beginning of interactive systems and is certainly not particular to sketching software.

Sketchpad, arguably the first sketching system, addressed this by letting the user control the mode with physical controls (buttons, toggle switches, dials) with the left hand [161]. GRAIL users did not explicitly enter modes to edit text and graphics. Instead, the meaning of user input was inferred by analyzing ink and its context [39]. These two early systems represent opposite extremes in ways to address the mode problem. Sketchpad's solution was explicit mode changes using nonpen input; GRAIL's solution was implicit mode changes done only with pen input.

There seems to be no clear "right" way to address the mode problem. Implicit mode changes may seem more natural, but only if the system correctly recognizes the user's intention. Recognition techniques are error-prone. Many systems therefore provide a combination of these two ways or impose drawing conventions.

Saund and Lank explored automatically recognizing mode based on the user's input in context of what has already been drawn [142]. Their inferred-mode protocol specifies an approach for analyzing the pen's trajectory and determining if an action may be taken unambiguously. If the user's intention is ambiguous, a mediator (such as those demonstrated by BURLAP [104]) provides the user with methods to resolve ambiguity.

Li et al. [98] compared mode-switching techniques for pen-based user interfaces. These techniques included the pen's button, press and hold, using the nondominant hand to press a physical button, a novel pressure-based method, and using the eraser end of the stylus. Interestingly, using the nondominant hand to switch modes was the fastest, the least error-prone, and best-liked method.

The "press and hold" approach is also used by Schilit et al. [145] who call this a "dwell" gesture. Microsoft Windows for Tablet PCs uses a dwell gesture for invoking right-click menus.

Scriboli's delimiters address the mode problem by allowing users to seamlessly switch between which objects to operate on and which commands to invoke [68]. For sketch-based systems the mode problem arises partly because there are multiple types of pen input. Some 
ink is intended to stay on the page and represents words, pictures, or other model elements (model operations). Other pen input indicates selections or commands (environment operations).

Flow selection [76] allows users to seamlessly change modes from drawing to selecting with a dwell gesture. Subsequent operations (such as moving part of a line) are performed by moving the stylus without lifting up. In the example in Figure 5.4, the selection strength depends on distance to the place the user is pressing and how long the stylus has been held down. Selection strength is then used by subsequent operations such as moving or smoothing.

\subsection{Application Areas of Sketching}

\subsubsection{Problem Solving}

Sketching supports everyday problem solving. A homeowner may estimate financial figures on the back of an envelope when managing household funds. A college student may draw a dorm room floor plan with furniture in various configurations to determine what is possible and desirable. Pencil and paper support these quick calculations very well.

One problem solving domain addressed by sketching systems is mathematics. MathPad ${ }^{2}$ [94] and MathBrush [89] let students draw pictures of natural phenomena and relate them to equations. For example, a physical system involving a mass on a spring can be represented with a drawing as well as with an equation as in Figure 5.5. The drawing

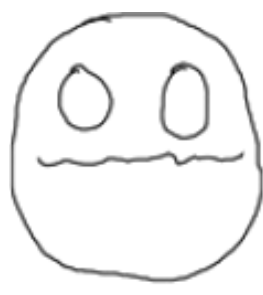

(a)

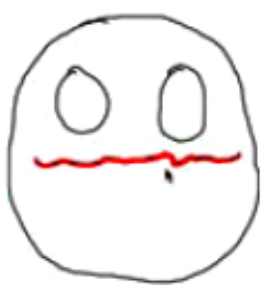

(b)

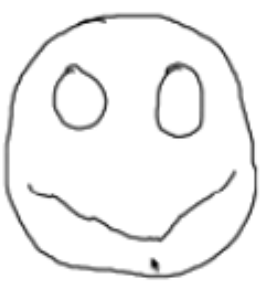

(c)

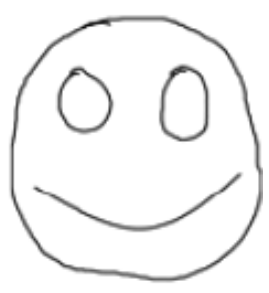

(d)

Fig. 5.4 Flow selection's mode changes done by alternately holding and moving the stylus [76]. Here the user positions the stylus near the middle of the figure's mouth and (b) moves it without lifting the pen (c). The user then holds the stylus still until the curve is smoothed (d) before completing the process by lifting the pen. 


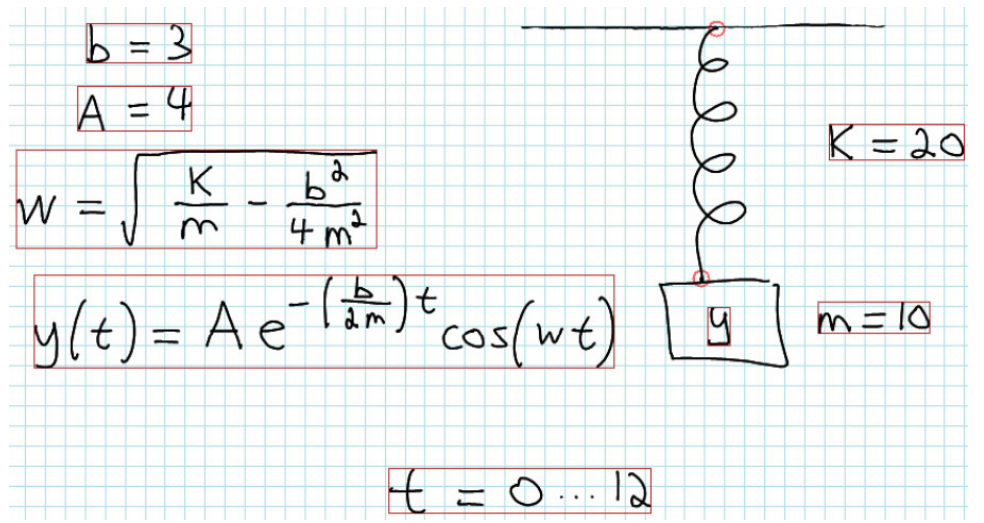

Fig. 5.5 A MathPad ${ }^{2}$ sketch showing hand-written equations corresponding to the drawing of the mass-and-spring system at right, which the system can animate.

directly communicates qualitative aspects about the system such as location and size of elements, while the equation governs the quantitative properties such as the weight's mass $m$, the spring constant $k$ and the time parameter's range $t$.

Many sketching systems allow users to simulate models. The MathPad $^{2}$ sketch in Figure 5.5 can be set into motion using the drawing as the initial condition, and the values $m, k$, and $t$ to control the animation.

Another example of problem solving addressed by sketching is editing and annotating written documents using ink based writing or gestures. XLibris is an electronic book that supports users in reading documents and keeping notes on the pages, which the system automatically organizes [145]. XLibris also recognizes certain ink commands as search requests. For example, say an XLibris user reading this paragraph circled, underlined, or highlighted the phrase "electronic book." This action silently triggers a database query. If a strong match is found, a link appears in the margin along with the word's definition.

\subsubsection{Sketch-Based Prototyping}

Sketching itself can be a form of prototyping. For example, user interface designers often build low-fidelity paper prototypes to use for 
cognitive walkthroughs or usabilty studies. The "sketchy" appearance may facilitate brainstorming or encourage people to make multiple interpretations of what the sketch means [36]. Alternately, designers may draw to unambiguously communicate specific designs [122].

Designers draw diagrams of how components fit together in order to better understand how to make things. At some point designers can move past sketching and build something - a user interface, a physical mechanism, a computer program. Before the final product is built, several prototypes are made to help clarify the problem domain, manufacturing constraints, usability issues, and so on. Designers often "go back to the drawing board," iteratively sketching and building prototypes.

Low-fidelity renderings of interfaces encourage discussion on the high-level functions the UI is intended to support. Conversely, highfidelity prototypes encourage discussion of details that are not important during brainstorming or early prototyping $[18,180]$.

SILK (Sketching Interfaces Like Krazy) allows designers to draw user interfaces and storyboards and then interact with them [92]. SILK recognizes sketches of a limited set of common user interface elements such as buttons, scroll bars, and text areas. It then transforms the sketch into a high-fidelity version of their drawn UI in the look-and-feel of their choice. The user may elect to retain the sketchy look and still interact with the recognized drawing. The sketch-to-prototype process is fast: users created an interface with SILK in one fifth of the time they needed to make the same interface with a structured interface builder.

DENIM is a system for prototyping web sites and individual page layouts [99]. DENIM allows designers to informally and incrementally create structures, first starting at one level of granularity, then moving up or down as appropriate. The ability to "zoom" from level to level is conducive to iterative web site design.

Other sketch-based systems have been developed for prototyping computer-based models including animations with K-Sketch, multimedia authoring with DEMAIS, and software development with MaramaSketch [16, 32, 61]. These tools allow designers to build prototypes or storyboards of dynamic systems by creating sketches according to conventional visual languages. 
One important property of physical sketches is their ability to record how a particular design evolved. Designers often keep journals of drawings and refer to them for reflection. Electronic sketching systems like SILK, ART019 [181], and NetDraw [135] support capturing and retrieving design histories of drawn objects.

\subsubsection{Sketching 3D Artifacts}

SILK, DENIM, and similar systems explored prototyping designs for electronic media. Another class of sketching systems supports rapid development of physical artifacts. Because physical objects are three dimensional, sketching systems targeting such output frequently involve 3D modeling.

SKETCH [182] is a 3D modeling system that accepts gestural input to perform modeling operations, rather than a traditional menu-based approach. The implementation described assumes a 3-button mouse as input, but the authors suggest that pen input would be better. The multiple buttons are used to address the mode problem, with shape operations performed with the left mouse button and camera operations with the right mouse button. SKETCH-N-MAKE, an "art to part" CAD system, added the ability to produce physical output [19].

SKETCH (and SKETCH-N-MAKE) define the characteristics of the model via a sequence of operations (extruding, cutting, etc.) on an existing model. This approach contrasts with a free-form drawing approach whereby designers draw shapes in 2D and the CAD system derives a 3D model. The free-form approach feels more "natural," approximating and augmenting pencil and paper.

Recently there has been progress in developing prototypes that construct 3D models based on freehand 2D sketches [100, 108]. These systems allow designers to directly specify shapes as they are conventionally drawn, rather than relying on mapping gestures to modeling commands.

Rapid prototyping machinery has become affordable. This gives people additional tools for making things. The Furniture Factory and Designosaur [124] projects are "sketch-to-fab" systems that enable people, even children, to design simple artifacts such as doll house furniture 
or dinosaur skeleton models. The Furniture Factory recognizes the 2D sketch as adjacent orthogonal planes, and selects appropriate jointing, leading to a CAD model suitable for manufacture on a laser cutter. Designosaur users sketch shapes of wooden "bones" and indicate locations for the bones to notch together.

\subsection{Sketching in Playful Applications}

Most of the systems described above have concerned the production of functional artifacts such as web sites, mechanisms or math equations. We can learn a lot (even about "serious" interactive systems) by building and evaluating systems that are playful.

ART019 [181] explores a novel sketch-based form of interaction for artists. It provides the ability to visualize drawings over time and easily combine model states from different moments in the drawing's creation history. For example, an artist may select a portion of a sketch by when strokes were applied, rather than where they were applied. DiFiore and Van Reeth [34] describe an interface for artistic sketch input for an animation tool that is as fluid as pencil-and-paper while giving animators the added ability to freely deform and edit drawings in an artful manner.

Crayon Physics Deluxe and Phun are aptly-named sketching programs wherein users draw shapes that are recognized as rigid bodies in a physical simulation $[37,85]$. The user controls game play by drawing shapes that interact with one another, subject to physical constraints such as gravity and rigid collisions (see Figure 5.6).

Paulson et al. [129] present a series of entertaining, educational sketch-based systems for children to learn by drawing. Their APPLES system lets users draw elements such as planets, black holes, and arrows representing initial velocity vectors. The recognized drawing then becomes alive, simulating gravitational pull and elastic collisions.

Another playful calligraphic application is Plushie, a system for designing stuffed toys [114]. Plushie users draw shapes that automatically turn into blobby, 3D objects rendered with cartoon-like, nonphotorealistic rendering. The system provides an intuitive drawing environment where people can make engaging, complex 3D models. 


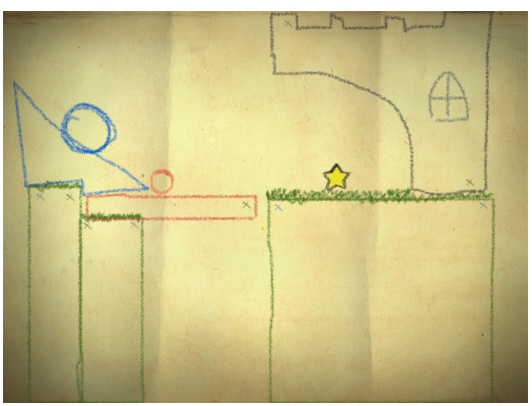

(a) Crayon Physics Deluxe

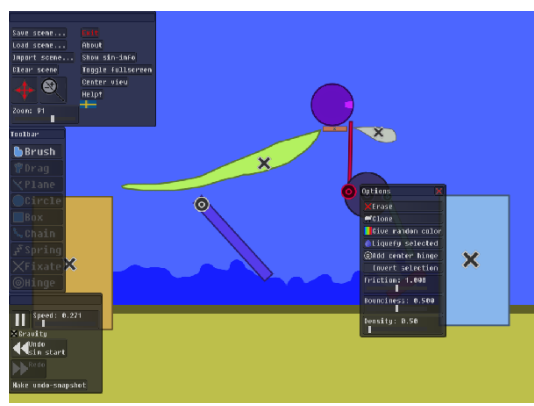

(b) Phun

Fig. 5.6 Two entertaining sketch-based physics simulation programs. Note the difference in how the programs render objects.

Like Plushie, FiberMesh builds on prior work by Igarashi and colleagues [73, 118]. FiberMesh enables users to edit 3D free-form models. Users enter one of several modes including deforming, rubbing, erasing, and type change. All strokes remain on the model, giving the user controls for later manipulation.

The "fun" applications presented here provide the basis for serious research. The act of drawing - even if it is a simple doodle - may help to clarify the designer's thoughts [29]. One participant in Nealen's informal user study said, "One great thing about this system is that one can start doodling without having a specific goal in mind, as if doodling on paper. One can just create something by drawing a stroke, and then gradually deform it guided by serendipity, which is very important for creative work [118]."

This section has discussed interaction topics of computational support for sketching. We began by presenting interaction concerning recognition. This includes ways for handling recognition errors, and how the system can react to sketch input and present interpretation results to the user. Several toolkits for developing sketch-based software were presented. Application areas for supporting human communication and design were described, with focus on areas where sketching is particularly useful. We discussed low-level interaction methods such as how users may select and act on objects, or how they may explicitly or implicitly enter modes. 


\section{6}

\section{Challenges and Opportunities}

In the preceding five sections we have reviewed work on computational support for sketching in design, beginning with GRAIL and Sketchpad in the 1960s through work that is going on today. We have viewed the field from various aspects: studies of "traditional" design sketching done on paper without benefit of computation; hardware that has been employed for computer supported sketching, techniques for, and management of sketch recognition; and interaction in sketch-based design software. Although we attempt in this review to cover the main themes in computer supported sketching for design, the field has grown large and diverse. We cannot hope to have captured all worthy and relevant work.

Our efforts in assembling this review began with a question: After forty years of research on computational support for sketching, why there are so few real world applications of this technology? Although the mouse has dominated computer interfaces since 1980, we see no sign that in daily life people are ceasing to draw with pencil and marker, on paper, and on whiteboards. Indeed, people draw on every available surface. We posit that if computational support for sketching really worked, it would be more widely adopted in a variety of applications 
and domains. As this has not yet happened, we asked ourselves: What is the state-of-the-art today, and what obstacles must be overcome before freehand sketching interaction will make its way from the research laboratory into the world of everyday use?

We found no simple single answer. However, our review of the literature in this field reveals research directions - and in some cases, challenges - in several areas that if resolved provides opportunities to develop successful real world applications. We summarize these below. Overall we maintain the optimistic outlook that the day of real-world sketching interfaces is still to come - whether just around the corner or a decade or more away. Each year we see a growing number of published papers and research projects. Hardware continues to advance, albeit somewhat more slowly. Further, the research community working on sketch-based interaction is growing both in size and in diversity of backgrounds.

\subsection{Future Work in Understanding Traditional Sketching}

Sketch-based software for design depends on research in two main areas. The first area is an understanding of the roles and uses of sketching in design: why, when, and how designers make quick drawings, and the role they play in the enterprise of designing. The second area is an understanding of the mechanics of sketching - how people make meaningful marks with a stylus. Research in these closely related topics can foster a better understanding of the computational mechanisms that can be brought to bear to support, in various ways, the processes of sketching for design.

\subsubsection{Sketching in Design}

The importance of sketching in design is asserted frequently in the literature, though few studies (including our own) go further than observing, as an argument for the authors' pen-based software project, that designers sketch. Yet, if we are to build sketching software that is truly useful for designers, we must gain a more systematic understanding of the ways that designers make diagrams, drawings, and sketches, how these representations serve design reasoning, how and what they 
communicate, and how they can be integrated with other knowledge and expertise. We cannot entirely separate the study of sketching as medium - from the study of design processes. Thus research in computer support for sketching can benefit by engaging designers and design researchers. Empirical studies of designers sketching, ethnographies, and analyses of user needs have already added to our knowledge. Nevertheless a thorough understanding of the functions of sketching in design will come only when we also engage designers in our quest.

\subsubsection{Mechanics of Sketching}

The mechanics of drawing and sketching is the second area where advances in research will lead to real world applications. There is a small but fascinating body of literature on how people draw things: we mentioned van Sommers' studies and there are others. Previously, film and video has been used to look closely at drawing mechanics. Now, pen technologies can fuel empirical studies on drawing ergonomics using Anoto, Wacom, or similar capture devices. These technologies can record drawing behavior that may be analyzed to better understand patterns and preferences for certain kinds of drawing tasks. What sequence do people choose in making a drawing, and what governs this choice? What, if any, is the relationship between pen speed, pressure, and the designer's level of confidence or certainty in the drawing? Answering these and other questions about drawing mechanics can lead, among other things, to software that can more effectively use these input data in building a more accurate model of the designer's actions.

Better knowledge about drawing mechanics can also inform the development of input and output hardware for sketching in design. We have seen hardware platforms move from light pens and CRTs, to expensive tethered digitizing surfaces, to low-cost tablets integrated with displays, digitizing whiteboards, and most recently e-ink and electronic paper. For input technologies, costs drop as resolution, reliability, and portability improve. Output technologies also improve in resolution, color capability, power consumption, and readability under diverse lighting conditions. The relatively recent introduction of Anoto's technology, introduced in 1998, reminds us that radically 
different approaches in hardware are possible and can lead to quite different applications. Two-handed interaction [88] is valuable in certain tasks, as is the ability to draw on arbitrary surfaces or in space [146]. It is easy to underestimate the effects of hardware on the performance of sketching systems: subtle ergonomic factors such as display screen parallax or the pen's feel on the drawing surface contribute to user satisfaction. Designers want to be comfortable drawing for extended periods of time, and even casual users are surprisingly sensitive to pen and surface ergonomics.

Under the broad rubric of computational mechanisms to support sketching in design, we have looked at (in Section 4) managing and integrating recognition into sketch-based interfaces, and (in Section 5) interaction techniques specific to sketching.

\subsection{Future Work in Computational Support for Sketching}

\subsubsection{Recognition}

Sketch recognition is a cousin to speech and gesture recognition, and there are issues common to all recognition-based interaction, for example segmentation, grouping, and managing conflicts, correction, con-

text, and $n$-best lists. Generally, the sketch recognition community could benefit from a close comparative study of research on recognition in these other modalities. We found no empirical data on what accuracy rate is acceptable in sketch recognition: How good must a recognizer be? Although this is likely to vary according to user and circumstance, the field would benefit by having benchmark data on acceptable accuracy in sketch recognition, similar to studies of acceptance of handwriting recognition accuracy.

Recognizer training is another area where research could advance support for sketching. Users are reluctant to devote time to training a recognizer, and so methods for incorporating training into ordinary use scenarios would be advantageous. Machine learning techniques might be applied to extract patterns from sketch data obtained from large numbers of users. When is it necessary to train a recognizer for individual users; when can a standard scheme serve all users? And when training is necessary, what interfaces are users most willing to tolerate? How 
can the number of needed training samples be minimized? Comparative usability and performance evaluations of training methods could be helpful.

Also related to recognition, when and under what circumstances should an application attempt recognition? Just how eager or lazy should a recognition engine be? When to be eager; when to be lazy? What heuristics can an application use to determine its recognition strategies? To what extent, and using what methods, can a system's knowledge of context support recognition? And finally, although many different approaches to recognition have been pursued, the challenge of recognizing sketches, whether domain-centric or domain-independent, remains an open problem.

A special (but important) case of sketch recognition is the problem of generating 3D models from 2D sketches. Again, many efforts have tackled particular kinds of 2D-to-3D sketch recognition. Yet specific constraints bound the capabilities of each of these projects: some systems make assumptions of orthonormal geometry; others handle curved surfaces. We are still far from a general purpose system for generating 3D models from sketches. The challenges here include dealing with incomplete drawings (e.g. lines truncated by the edge of the sketch); incorrect drawings (not made to correct isometry or perspective); the use of shading, hatching, and line weight to inform model-construction. Here we also distinguish between projects that aim to recognize and parse projection drawings made according to the traditional conventions, and those (like SKETCH [182] and SketchUp [54]) that use an artificial language of gestural commands to construct models.

\subsubsection{Interaction Techniques}

Another broad area where research can advance software support for sketching in design is interaction techniques specifically tuned for the pen. The early work at PARC that produced the WIMP interface led to widely adopted conventions for using the mouse to interact with applications. We have yet to see a similar set of conventions that emerge for using the pen to interact with applications, though as we saw in

Section 5, some work has been done along these lines. Pen interaction 
techniques might leverage input data such as pressure [136] and pen angle, in addition to the customary position, timing, and pen-up, pendown events. It may be fruitful also to look at the design of screen widgets specifically for use with the pen, rather than assuming that the widgets that work for the mouse are equally suited for pen-based interaction. And work with gestures such as the "pigtail" or the technique of "crossing" screen items suggests that developing and adopting a conventional language of pen gestures may be helpful [9, 68].

Stepping up a level from the pen and screen, support for sketching will require other interaction techniques. For example, managing recognition errors and ambiguities in a sketching program without unduly distracting the designer remains a challenge. Research on interruption management may be useful: the system could assess the user's state and decide when is an appropriate time to query for resolving an error, conflict, or ambiguity.

\subsubsection{Application Architectures}

Perhaps one of the greatest obstacles to widespread adoption of sketching interaction is the underlying architecture of application software and the software engineering assumptions that this entails. A strength of sketching is that it can convey decisions on a spectrum from ambiguity and vagueness to precision and certainty. However, as long as the underlying software does not support such a spectrum, it will be difficult to take advantage of this feature. That is, whatever degree of ambiguity or precision the user may wish to convey through the sketch will be resolved before the application gets hold of it. In this review, we have touched many times on the idea that sketches can capture the user's level of commitment and precision, but until application software can use this information, the system's capacity to capture and convey it is wasted. Here we contrast speech and sketch recognition: It is a fair assumption that a speaker has intended to make a specific and precise utterance, and it is the job of the listener to recognize what that was. As we have pointed out, in design this is not always the case.

Sketching, however, does not only happen at the early stages of designing. Designers sketch throughout the process, and so software 
must also be able to capture and use sketch input even during later stage design. The earliest, exploratory sketches may convey a wide range of alternatives quickly. Drawings made in concluding phases resolve detail and entail highly precise decision making. Effective sketch-based applications must be able to support designers throughout this process.

In the vein of innovative application architectures that take advantage of sketching input, it is worth remarking that many of the innovations in Sketchpad were programming language ideas, not what we would now see as HCI ideas. The software architecture of Sketchpad a constraint solver at its core, an object-like representation of prototypes and instances - these were deeply integrated into the design of the program. It may take more than wrapping existing applications with a "sketching interface" in order to reap the advantages of penbased interaction.

\subsubsection{Toolkits}

Most of the systems we reviewed here were built from scratch, using only the most basic device and graphics libraries. Building from the ground up can allow more divergent and potentially innovative work. However, this comes at a heavy cost: a great deal of redundant effort is spent by the sketching research community building similar lowlevel functionality. In order to advance the field and build more robust and sharable sketching applications, programmers can rely on toolkits and libraries to perform many of the mundane and common tasks. Several toolkits have been proposed and developed (e.g. SATIN and InkKit $[69,133])$ but these have not gained widespread acceptance. It would be worth looking into why more researchers and developers have not adopted these toolkits. What would be the required characteristics of a successful toolkit for sketching - both research prototypes and full-fledged applications - remains an open question.

\subsection{Conclusion: In Support of Visual Thinking}

Computational support for sketching has a long and interesting history

dating back to the early days of computing. Some of the first graphical 
interfaces in the 1960s demonstrated compelling features that even today are not wholly integrated into pen computing. Despite advances in hardware (digitizing whiteboards, the Wacom ${ }^{\mathrm{TM}}$ [173] and Anoto pen technologies, the Tablet PC) sketching remains a niche area. Perhaps it always will. On the other hand, it may be that overcoming a number of obstacles would make pen-based sketch interaction far more attractive in a range of application areas. People, after all, still draw.

Certainly for designers - and by this we mean not only those in the "artistic" or "creative" professions but also chemical, electrical, mechanical engineers, economists, anyone who uses graphical representations in their work - better computer support for sketching would be a boon. Arguably, sketching and diagramming is not just a matter of recording geometry of ideas that have been worked out in the head. Rather, as Arnheim put it, drawing is a medium for visual thinking [11]. Seen in this way, supporting sketching for design goes beyond strategies for more accurately capturing pen strokes or ink, or tuning recognition. Truly inspired work on computational support for sketching will see its task as supporting designers - and all users - in thinking visually. 


\section{References}

[1] J. Accot and S. Zhai, "More than dotting the i's - foundations for crossingbased interfaces," in CHI '02: Proceedings of the SIGCHI Conference on Human Factors in Computing Systems, pp. 73-80, New York, NY, USA: ACM, 2002.

[2] C. Alvarado, "A natural sketching environment: Bringing the computer into early stages of mechanical design," Master's thesis Massachusetts Institute of Technology, 2000.

[3] C. Alvarado, "Sketch recognition user interfaces: Guidelines for design and development," in Proceedings of AAAI Fall Symposium on Intelligent Penbased Interfaces, 2004.

[4] C. Alvarado and R. Davis, "SketchREAD: A multi-domain sketch recognition engine," in UIST '04: Proceedings of the 17th Annual ACM Symposium on User Interface Software and Technology, 2004.

[5] C. Alvarado and R. Davis, "Dynamically constructed bayes nets for multidomain sketch understanding," in International Joint Conference on Artificial Intelligence, 2005.

[6] F. Anastacio, M. C. Sousa, F. Samavati, and J. A. Jorge, "Modeling plant structures using concept sketches," in NPAR '06: Proceedings of the 4th International Symposium on Non-photorealistic Animation and Rendering, pp. 105-113, New York, NY, USA: ACM, 2006.

[7] Anoto, "Development guide for service enabled by anoto functionality," Technical report, Anoto AB, 2002.

[8] L. Anthony, J. Yang, and K. R. Koedinger, "Evaluation of multimodal input for entering mathematical equations on the computer," in CHI '05: $\mathrm{CHI}$ '05 Extended Abstracts on Human Factors in Computing Systems, pp. 1184-1187, New York, NY, USA: ACM, 2005. 
[9] G. Apitz and F. Guimbretière, "CrossY: A crossing-based drawing application," in UIST '04: Proceedings of the 17th Annual ACM symposium on User Interface Software and Technology, pp. 3-12, New York, NY, USA: ACM, 2004.

[10] Apple Inc., "Apple Inkwell," http://www.apple.com/sg/macosx/features/ inkwell/, 2007.

[11] R. Arnheim, Visual Thinking. London: Faber and Faber, 1969.

[12] J. Arnowitz, M. Arent, and N. Berger, Effective Prototyping for Software Makers. Morgan Kaufmann, 2006.

[13] J. Arvo and K. Novins, "Appearance-preserving manipulation of hand-drawn graphs," in GRAPHITE '05: Proceedings of the 3rd International Conference on Computer Graphics and Interactive Techniques in Australasia and South East Asia, pp. 61-68, New York, NY, USA: ACM, 2005.

[14] Autodesk Inc., "Autodesk Maya," 2008. http://autodesk.com.

[15] S.-H. Bae, R. Balakrishnan, and K. Singh, "ILoveSketch: As-natural-aspossible sketching system for creating 3D curve models," in Proceedings of UIST'08 (to appear), 2008.

[16] B. P. Bailey and J. A. Konstan, "Are informal tools better?: comparing DEMAIS, pencil and paper, and authorware for early multimedia design," in CHI '03: Proceedings of the SIGCHI Conference on Human Factors in Computing Systems, pp. 313-320, New York, NY, USA: ACM, 2003.

[17] O. Bimber, L. M. Encarnacao, and A. Stork, "A multi-layered architecture for sketch-based interaction within virtual environments," Computers and Graphics, vol. 24, pp. 851-867, 2000.

[18] A. Black, "Visible planning on paper and on screen: The impact of working medium on decision-making by novice graphic designers," Behaviour and Information Technology, vol. 9, no. 4, pp. 283-296, 1990.

[19] M. Bloomenthal, R. Zeleznik, R. Fish, L. Holden, A. Forsberg, R. Riesenfeld, M. Cutts, S. Drake, H. Fuchs, and E. Cohen, "SKETCH-N-MAKE: Automated machining of CAD sketches," in Proceedings of ASME DETC'98, pp. 1-11, 1998.

[20] D. Blostein, E. Lank, A. Rose, and R. Zanibbi, "User interfaces for on-line diagram recognition," in GREC '01: Selected Papers from the Fourth International Workshop on Graphics Recognition Algorithms and Applications, pp. 92-103, London, UK: Springer-Verlag, 2002.

[21] B. Buxton, Sketching User Experiences. Morgan Kaufmann Publishers, 2007.

[22] X. Chen, S. B. Kang, Y.-Q. Xu, J. Dorsey, and H.-Y. Shum, "Sketching reality: Realistic interpretation of architectural designs," ACM Transations Graphics, vol. 27 , no. 2 , pp. 1-15, 2008.

[23] M. B. Clowes, "On seeing things," Artificial Intelligence, vol. 2, pp. 79-116, 1971.

[24] J. M. Cohen, L. Markosian, R. C. Zeleznik, J. F. Hughes, and R. Barzel, "An interface for sketching 3D curves," in I3D '99: Proceedings of the 1999 Symposium on Interactive $3 D$ Graphics, pp. 17-21, New York, NY, USA: ACM, 1999. 
[25] R. Cole, J. Mariani, H. Uszkoreit, A. Zaenen, and V. Zue, Survey of the State of the Art in Human Language Technology. Center for Spoken Language Understanding CSLU, Carnegie Mellon University, 1995.

[26] G. Costagliola, V. Deufemia, F. Ferrucci, and C. Gravino, "Exploiting XPG for visual languages definition, analysis and development," Electronic Notes in Theoretical Computer Science, vol. 82, no. 3, pp. 612-627, 2003.

[27] G. Costagliola, V. Deufemia, and M. Risi, "Sketch grammars: A formalism for describing and recognizing diagrammatic sketch languages," in International Conference on Document Analysis and Recognition, 2005.

[28] N. Cross, "The nature and nurture of design ability," Design Studies, vol. 11, no. 3, pp. 127-140, 1990.

[29] N. Cross, "Natural intelligence in design," Design Studies, vol. 20, no. 1, pp. 25-39, 1999.

[30] Cross Pen Computing Group, "CrossPad," 1998. Portable digital notepad.

[31] R. Davis, "Sketch understanding: Toward natural interaction toward natural interaction," in SIGGRAPH '06: ACM SIGGRAPH 2006 Courses, p. 4, New York, NY, USA: ACM, 2006.

[32] R. C. Davis, B. Colwell, and J. A. Landay, "K-sketch: A 'kinetic' sketch pad for novice animators," in CHI '08: Proceeding of the Twenty-Sixth Annual SIGCHI Conference on Human Factors in Computing Systems, pp. 413-422, New York, NY, USA: ACM, 2008.

[33] P. de Bruyne, "Acoustic radar graphic input device," in SIGGRAPH '80: Proceedings of the 7th Annual Conference on Computer Graphics and Interactive Techniques, pp. 25-31, New York, NY, USA: ACM, 1980.

[34] F. Di Fiore and F. V. Reeth, "A multi-level sketching tool for pencil-and-paper animation," in Sketch Understanding: Papers from the 2002 American Association for Artificial Intelligence (AAAI 2002) Spring Symposium, pp. 32-36, 2002.

[35] E. Y.-L. Do, The Right Tool at the Right Time: Investigation of Freehand Drawing as an Interface to Knowledge Based Design Tools. PhD thesis, Georgia Institute of Technology, 1998.

[36] E. Y.-L. Do, "Design sketches and sketch design tools," Knowledge-Based Systems, vol. 18, no. 8, pp. 838-405, 2005.

[37] E. Ernerfeldt, (Forthcoming), "MS Thesis on Phun," Master's thesis, Umeå University, 2008.

[38] Electronic Arts Inc., "The Sims," 2008. http://thesims.ea.com.

[39] T. O. Ellis, J. F. Heafner, and W. L. Sibley, "The GRAIL Project: An experiment in man-machine communications," Technical report, RAND Memorandum RM-5999-ARPA, RAND Corporation, 1969.

[40] M. Fonseca and J. Jorge, "Using fuzzy logic to recognize geometric shapes interactively," The Ninth IEEE International Conference on Fuzzy Systems, 2000. FUZZ IEEE 2000, vol. 1, pp. 291-296, 2000.

[41] M. Fonseca, C. Pimentel, and J. Jorge, "CALI: An online scribble recognizer for calligraphic interfaces," in AAAI 2002 Spring Symposium (Sketch Understanding Workshop), pp. 51-58, 2002. 
[42] K. D. Forbus, "Exploring spatial cognition through sketch understanding," in Spatial Cognition, http://conference.spatial-cognition.de/sc08/tutorials/T-1, 2008.

[43] K. D. Forbus, J. Usher, and V. Chapman, "Sketching for military courses of action diagrams," in Proceedings of Intelligent User Interfaces '03, 2003.

[44] C. Frankish, R. Hull, and P. Morgan, "Recognition accuracy and user acceptance of pen interfaces," in CHI '95: Proceedings of the SIGCHI Conference on Human Factors in Computing Systems, pp. 503-510, New York, NY, USA: ACM Press/Addison-Wesley Publishing Co., 1995.

[45] R. Futrelle, "Ambiguity in visual language theory and its role in diagram parsing," Proceedings. 1999 IEEE Symposium on Visual Languages, 1999, pp. 172175, 1999.

[46] R. P. Futrelle and N. Nikolakis, "Efficient analysis of complex diagrams using constraint-based parsing," in Proceedings of the Third International Conference on Document Analysis and Recognition (ICDAR'95), 1995.

[47] J. Geißler, "Gedrics: The next generation of icons," in Proceedings of the 5th International Conference on Human-Computer Interaction (INTERACT'95), 1995.

[48] L. Gennari, L. B. Kara, and T. F. Stahovich, "Combining geometry and domain knowledge to interpret hand-drawn diagrams," Computers and Graphics, vol. 29, no. 4, pp. 547-562, 2005.

[49] J. Glasgow, N. H. Narayanan, and B. Chandrasekaran, eds., Diagrammatic Reasoning: Cognitive and Computational Perspectives. MIT Press, 1995.

[50] V. Goel, Sketches of Thought. Cambridge, MA: MIT Press/A Bradford Book, 1995.

[51] G. Goldschmidt, "The dialectics of sketching," Creativity Research journal, vol. 4, no. 2, pp. 123-143, 1991.

[52] G. Goldschmidt, "The backtalk of self-generated sketches," in Spatial and Visual Reasoning in Design, Syndey, Australia: Key Center of Design Computing, 1999.

[53] N. Goodman, Languages of Art: An Approach to a Theory of Symbols. Indianapolis, Indiana: Hackett, Second ed., 1976.

[54] Google Inc., "Google Sketchup," 2008. http://www.sketchup.com/.

[55] I. J. Grimstead and R. R. Martin, "Creating solid models from single 2D sketches," in SMA '95: Proceedings of the Third ACM Symposium on Solid Modeling and Applications, pp. 323-337, New York, NY, USA: ACM, 1995.

[56] G. F. Groner, "Real-time recognition of handprinted text," Technical report, RM-5016-ARPA, RAND Corporation, 1966.

[57] M. D. Gross, "Stretch-a-sketch, a dynamic diagrammer," in Proceedings of IEEE Symposium on Visual Languages and Human-Centric Computing, pp. 232-238, 1994.

[58] M. D. Gross, "The electronic cocktail napkin: A computational environment for working with design diagrams," Design Studies, vol. 17, no. 1, pp. 53-69, 1996.

[59] M. D. Gross and E. Y.-L. Do, "Ambiguous intentions: A paper-like interface for creative design," in UIST '04: ACM Conference on User Interface Software Technology, pp. 183-192, Seattle, WA, 1996. 
[60] M. D. Gross and E. Y.-L. Do, "Drawing on the back of an envelope," Computers and Graphics, vol. 24, no. 6, pp. 835-849, 2000.

[61] J. Grundy and J. Hosking, "Supporting generic sketching-based input of diagrams in a domain-specific visual language meta-tool," in ICSE '07: International Conference on Software Engineering, pp. 282-291, Washington, DC: IEEE Computer Society, 2007.

[62] F. Guimbretière, "Paper augmented digital documents," in UIST '03: Proceedings of the 16th Annual ACM Symposium on User Interface Software and Technology, pp. 51-60, New York, NY, USA: ACM, 2003.

[63] T. Hammond and R. Davis, "LADDER, a sketching language for user interface developers," Elsevier, Computers and Graphics, vol. 29, pp. 518-532, 2005.

[64] T. Hammond and R. Davis, "Interactive learning of structural shape descriptions from automatically generated near-miss examples," in Intelligent User Interfaces (IUI), pp. 37-40, 2006.

[65] J. Y. Han, "Low-cost multi-touch sensing through frustrated total internal reflection," in UIST '05: Proceedings of the 18th Annual ACM Symposium on User Interface Software and Technology, pp. 115-118, New York, NY, USA: ACM, 2005.

[66] D. Hendry, "Sketching with conceptual metaphors to explain computational processes," in IEEE Symposium on Visual Languages/Human-Centric Computing, pp. 95-102, Brighton, UK: IEEE Computer Society Press, 2006.

[67] K. Hinckley, "Input technologies and techniques," in Handbook of HumanComputer Interaction, (A. Sears and J. A. Jacko, eds.), Lawrence Erlbaum and Associates, 2006.

[68] K. Hinckley, P. Baudisch, G. Ramos, and F. Guimbretiere, "Design and analysis of delimiters for selection-action pen gesture phrases in scriboli," in CHI '05: Proceedings of the SIGCHI Conference on Human Factors in Computing Systems, pp. 451-460, New York, NY, USA: ACM, 2005.

[69] J. Hong and J. Landay, "SATIN: A toolkit for informal ink-based applications," CHI Letters (13th Annual ACM Symposium on User Interface Software and Technology: UIST 2000), vol. 2, no. 2, pp. 63-72, 2000.

[70] J. Hong, J. Landay, A. C. Long, and J. Mankoff, "Sketch recognizers from the end-user's the designer's and the programmer's perspective," in AAAI Spring Symposium on Sketch Understanding, (T. Stahovic, J. Landay, and R. Davis, eds.), Menlo Park, CA: AAAI Press, 2002.

[71] D. A. Huffman, "Impossible objects as nonsense sentences," Machine Intelligence, vol. 6, pp. 295-323, 1971.

[72] T. Igarashi and J. F. Hughes, "A suggestive interface for 3D drawing," in UIST '01: Proceedings of the 14th Annual ACM Symposium on User Interface Software and Technology, pp. 173-181, New York, NY, USA: ACM, 2001.

[73] T. Igarashi and J. F. Hughes, "Smooth meshes for sketch-based freeform modeling," in I3D '03: Proceedings of the 2003 Symposium on Interactive 3D Graphics, pp. 139-142, New York, NY, USA: ACM, 2003.

[74] T. Igarashi, S. Matsuoka, S. Kawachiya, and H. Tanaka, "Interactive beautification: A technique for rapid geometric design," in UIST '97: Proceedings of the 10th Annual ACM Symposium on User Interface Software and Technology, pp. 105-114, New York, NY, USA: ACM, 1997. 
[75] T. Igarashi, S. Matsuoka, and H. Tanaka, "Teddy: A sketching interface for 3D freeform design," in ACM SIGGRAPH'99, pp. 409-416, Los Angeles, California, 1999.

[76] G. Johnson, M. D. Gross, and E. Y.-L. Do, "Flow selection: A time-based selection and operation technique for sketching tools," in 2006 Conference on Advanced Visual Interfaces, pp. 83-86, Venice, Italy, 2006.

[77] W. Ju, A. Ionescu, L. Neeley, and T. Winograd, "Where the wild things work: Capturing shared physical design workspaces," in CSCW'04: Proceedings of the 2004 ACM Conference on Computer Supported Cooperative Work, pp. 533-541, New York, NY, USA: ACM, 2004.

[78] G. Kanizsa, Organization in Vision: Essays on Gestalt Perception. Praeger, New York, 1979.

[79] L. B. Kara, C. M. D'Eramo, and K. Shimada, "Pen-based styling design of 3D geometry using concept sketches and template models," in SPM '06: Proceedings of the 2006 ACM Symposium on Solid and Physical Modeling, pp. 149-160, New York, NY, USA: ACM, 2006.

[80] L. B. Kara and T. F. Stahovich, "An image-based, trainable symbol recognizer for hand-drawn sketches," Computers and Graphics, vol. 29, no. 4, pp. 501-517, 2005.

[81] M. Karam and M. C. Schraefel, "Investigating user tolerance for errors in vision-enabled gesture-based interactions," in AVI '06: Proceedings of the Working Conference on Advanced Visual Interfaces, pp. 225-232, New York, NY, USA: ACM, 2006.

[82] O. A. Karpenko and J. F. Hughes, "SmoothSketch: 3D free-form shapes from complex sketches," ACM Transactions on Graphics, vol. 25, no. 3, pp. 589$598,2006$.

[83] A. Kay, "Alan Kay lecture on early interactive computer systems," http://www.newmediareader.com/cd_samples/Kay/index.html, 1986.

[84] D. H. Kim and M.-J. Kim, "A curvature estimation for pen input segmentation in sketch-based modeling," Computer-Aided Design, vol. 38, no. 3, pp. 238$248,2006$.

[85] kloonigames.com, "Crayon physics deluxe," http://www.kloonigames.com/ crayon/, 2008.

[86] K. Kuczun and M. D. Gross, "Local area network tools and tasks," in ACM Conference on Designing Interactive Systems, pp. 215-221, 1997.

[87] G. Kurtenbach and W. Buxton, "Issues in combining marking menus and direct manipulation techniques," in Symposium on User Interface Software and Technology, pp. 137-144, ACM, 1991.

[88] G. Kurtenbach, G. Fitzmaurice, T. Baudel, and B. Buxton, "The design of a GUI paradigm based on tablets, two-hands, and transparency," in CHI '97: Proceedings of the SIGCHI Conference on Human Factors in Computing Systems, pp. 35-42, New York, NY, USA: ACM, 1997.

[89] G. Labahn, S. MacLean, M. Marzouk, I. Rutherford, and D. Tausky, "A preliminary report on the MathBrush pen-math system," in Maple 2006 Conference, pp. 162-178, 2006. 
[90] F. Lakin, J. Wambaugh, L. Leifer, D. Cannon, and C. Sivard, "The electronic design notebook: Performing medium and processing medium," Visual Computer: International Journal of Computer Graphics, vol. 5, no. 4, 1989.

[91] M. LaLomia, "User acceptance of handwritten recognition accuracy," in CHI '94: Conference Companion on Human Factors in Computing Systems, pp. 107-108, New York, NY, USA: ACM, 1994.

[92] J. A. Landay, "SILK: Sketching interfaces like krazy," in ACM CHI 1996, pp. 398-399, Vancouver, Canada, 1996.

[93] J. Larkin and H. Simon, "Why a diagram is (Sometimes) worth ten thousand words," Cognitive Science Journal, vol. 11, pp. 65-99, 1987.

[94] J. LaViola and R. C. Zeleznik, "MathPad": A system for the creation and exploration of mathematical sketches," ACM Transactions on Graphics, vol. 23, no. 3, pp. 432-440, 2004.

[95] J. Lee, S. Hudson, and P. Dietz, "Hybrid infrared and visible light projection for location tracking," in UIST '0\%: Proceedings of the 20th Annual ACM Symposium on User Interface Software and Technology, pp. 57-60, New York, NY, USA: ACM, 2007.

[96] J. C. Lee, "Projector-based location discovery and tracking," PhD thesis, Carnegie Mellon University, 5000 Forbes Ave, Pittsburgh PA, USA, 2008.

[97] W. Lee, L. B. Kara, and T. F. Stahovich, "An efficient graph-based symbol recognizer," in EUROGRAPHICS Workshop on Sketch-Based Interfaces and Modeling, (T. F. Stahovich and M. C. Sousa, eds.), 2006.

[98] Y. Li, K. Hinckley, Z. Guan, and J. A. Landay, "Experimental analysis of mode switching techniques in pen-based user interfaces," in CHI 2005, 2005.

[99] J. Lin, M. Newman, J. Hong, and J. Landay, "DENIM: Finding a tighter fit between tools and practice for web site design," in CHI Letters, pp. 510-517, 2000.

[100] H. Lipson and M. Shpitalni, "Correlation-based reconstruction of a 3D object from a single freehand sketch," in AAAI 2002 Spring Symposium (Sketch Understanding Workshop), 2002.

[101] J. Lladós, E. Martí, and J. J. Villanueva, "Symbol recognition by error-tolerant subgraph matching between region adjacency graphs," IEEE Transactions on Pattern Analysis and Machine Intelligence, vol. 23, no. 10, pp. 1137-1143, 2001.

[102] A. C. Long, J. A. Landay, L. A. Rowe, and J. Michiels, "Visual similarity of pen gestures," in CHI '00: Proceedings of the SIGCHI Conference on Human Factors in Computing Systems, pp. 360-367, New York, NY, USA: ACM, 2000.

[103] J. V. Mahoney and M. P. J. Fromherz, "Three main concerns in sketch recognition and an approach to addressing them," in Sketch Understanding, Papers from the 2002 AAAI Spring Symposium, 2002.

[104] J. Mankoff, "Providing integrated toolkit-level support for ambiguity in recognition-based interfaces," in CHI '00: CHI '00 Extended Abstracts on Human Factors in Computing Systems, pp. 77-78, New York, NY, USA: ACM, 2000. 
[105] J. Mankoff, G. D. Abowd, and S. E. Hudson, "OOPS: A toolkit supporting mediation techniques for resolving ambiguity in recognition-based interfaces," Computers and Graphics, vol. 24, no. 6, pp. 819-834, 2000.

[106] D. Marr, "Early processing of visual information," Philosophical Transactions of the Royal Society of London B, vol. 275, pp. 483-519, 1976.

[107] J. Mas, G. Sánchez, and J. Lladós, "An adjacency grammar to recognize symbols and gestures in a digital pen framework," in Pattern Recognition and Image Analysis, pp. 115-122, Springer, 2005.

[108] M. Masry, D. Kang, and H. Lipson, "A freehand sketching interface for progressive construction of 3D objects," Computers and Graphics, vol. 29, no. 4, pp. 563-575, 2005.

[109] A. Meyer, "Pen computing: A technology overview and a vision," SIGCHI Bulletin, vol. 27, no. 3, pp. 46-90, 1995.

[110] Microsoft Inc., "Surface," http://www.microsoft.com/surface/, 2007.

[111] J. Mitani, H. Suzuki, and F. Kimura, "3D sketch: Sketch-based model reconstruction and rendering," in IFIP Workshop Series on Geometric Modeling: Fundamentals and Applications, Parma, Italy, 2000.

[112] T. P. Moran, E. Saund, W. van Melle, A. U. Gujar, K. P. Fishkin, and B. L. Harrison, "Design and technology for collaborage: Collaborative collages of information on physical walls," CHI Letters, vol. 1, no. 1, 1999.

[113] T. P. Moran, W. van Melle, and P. Chiu, "Spatial interpretation of domain objects integrated into a freeform electronic whiteboard," in Proceedings of UIST'98, 1998.

[114] Y. Mori and T. Igarashi, "Plushie: An interactive design system for plush toys," in Proceedings of SIGGRAPH 200\%, ACM, 2007.

[115] D. Mumford, "Elastica and computer vision," in Algebraic Geometry and its Applications, (C. L. Bajaj, ed.), Springer-Verlag, New York, 1994.

[116] B. Myers, S. Y. Park, Y. Nakano, G. Mueller, and A. Ko, "How designers design and program interactive behaviors," in Proceedings of IEEE Symposium on Visual Languages and Human-Centric Computing, (P. Bottoni, M. B. Rosson, and M. Minas, eds.), pp. 177-184, 2008.

[117] E. D. Mynatt, T. Igarashi, W. K. Edwards, and A. LaMarca, "Flatland: New dimensions in office whiteboards," in CHI '99: Proceedings of the SIGCHI Conference on Human Factors in Computing Systems, pp. 346-353, New York, NY, USA: ACM, 1999.

[118] A. Nealen, T. Igarashi, O. Sorkine, and M. Alexa, "FiberMesh: Designing freeform surfaces with 3D Curves," in ACM SIGGRAPH 200\%, San Diego, CA: ACM Transactions on Computer Graphics, 2007.

[119] A. Nealen, O. Sorkine, M. Alexa, and D. Cohen-Or, "A sketch-based interface for detail-preserving mesh editing," in SIGGRAPH '05: ACM SIGGRAPH 2005 Papers, pp. 1142-1147, New York, NY, USA: ACM, 2005.

[120] N. Negroponte, Soft Architecture Machines. Cambridge, MA: MIT Press, 1975.

[121] B. Neiman, E. Y.-L. Do, and M. D. Gross, "Sketches and their functions in early design: A retrospective analysis of two houses," in Design Thinking Research Symposium, 1999.

[122] M. W. Newman and J. A. Landay, "Sitemaps, storyboards, and specifications: A sketch of Web site design practice," in DIS '00: Proceedings of the 3rd 
Conference on Designing Interactive Systems, pp. 263-274, New York, NY, USA: ACM, 2000.

[123] W. Newman and R. Sproull, Principles of Interactive Computer Graphics. McGraw-Hill, Second ed., 1979.

[124] Y. Oh, G. Johnson, M. D. Gross, and E. Y.-L. Do, "The designosaur and the furniture factory: Simple software for fast fabrication," in 2nd International Conference on Design Computing and Cognition (DCC06), 2006.

[125] S. Oviatt, A. Arthur, and J. Cohen, "Quiet interfaces that help students think," in UIST '06: Proceedings of the 19th Annual ACM Symposium on User Interface Software and Technology, pp. 191-200, New York, NY, USA: ACM, 2006.

[126] S. Oviatt and P. Cohen, "Multimodal processes that process what comes naturally," Communications on ACM, vol. 43, no. 3, pp. 45-53, 2000.

[127] Palm Inc., "Palm Digital PDA," http://www.palm.com/, 2007.

[128] B. Pasternak and B. Neumann, "Adaptable drawing interpretation using object-oriented and constraint-based graphic specification," in Proceedings of the International Conference on Document Analysis and Recognition (ICDAR '93), 1993.

[129] B. Paulson, B. Eoff, A. Wolin, A. Johnston, and T. Hammond, "Sketch-based educational games: "Drawing" kids away from traditional interfaces," in Interaction Design and Children (IDC 2008) (to appear), 2008.

[130] T. Pavlidis and C. J. V. Wyk, "An automatic beautifier for drawings and illustrations," SIGGRAPH Computer Graphics, vol. 19, no. 3, pp. 225-234, 1985.

[131] E. R. Pedersen, K. McCall, T. P. Moran, and F. G. Halasz, "Tivoli: An electronic whiteboard for informal workgroup meetings," in CHI '93: Proceedings of the INTERACT '93 and CHI '93 Conference on Human Factors in Computing Systems, pp. 391-398, New York, NY, USA: ACM, 1993.

[132] B. Plimmer, "Experiences with digital pen, keyboard and mouse usability," Journal on Multimodal User Interfaces, vol. 2, no. 1, pp. 13-23, July 2008.

[133] B. Plimmer and I. Freeman, "A toolkit approach to sketched diagram recognition," in Proceedings of HCI 2007, British Computer Society, 2007.

[134] G. Polya, How To Solve It. Princeton University Press, 1945.

[135] D. Qian and M. D. Gross, "Collaborative design with NetDraw," in Proceedings of CAAD Futures 1999 Conference, vol. CAAD Futures 'Computers in Building', 1999.

[136] G. Ramos, M. Boulos, and R. Balakrishnan, "Pressure widgets," in CHI '04: Proceedings of the SIGCHI Conference on Human Factors in Computing Systems, pp. 487-494, New York, NY, USA: ACM, 2004.

[137] H. Rittel and M. Webber, "Dilemmas in a general theory of planning," Policy Sciences, vol. 4, pp. 155-169, 1973.

[138] D. Rubine, "Specifying gestures by example," SIGGRAPH Computer Graphics, vol. 25, no. 4, pp. 329-337, 1991.

[139] P. Santos, A. J. Baltzer, A. N. Badre, R. L. Henneman, and M. Miller, "On handwriting recognition performance: Some experimental results," in Proceedings of the Human Factors Society 36th Annual Meeting, pp. 283-287, 1992. 
[140] E. Saund, "Bringing the marks on a whiteboard to electronic life," in CoBuild '99: Proceedings of the Second International Workshop on Cooperative Buildings, Integrating Information, Organization, and Architecture, pp. 69-78, London, UK: Springer-Verlag, 1999.

[141] E. Saund, D. Fleet, D. Larner, and J. Mahoney, "Perceptually-supported image editing of text and graphics," in UIST '03: Proceedings of the 16th Annual ACM Symposium on User Interface Software and Technology, pp. 183192, New York, NY, USA: ACM, 2003.

[142] E. Saund and E. Lank, "Stylus input and editing without prior selection of mode," in UIST '03: Proceedings of the 16th Annual ACM Symposium on User Interface Software and Technology, pp. 213-216, New York, NY, USA: ACM, 2003.

[143] E. Saund, J. Mahoney, D. Fleet, D. Larner, and E. Lank, "Perceptual organization as a foundation for intelligent sketch editing," in AAAI Spring Symposium on Sketch Understanding, pp. 118-125, American Association for Artificial Intelligence, 2002.

[144] E. Saund and T. P. Moran, "A perceptually-supported sketch editor," in $A C M$ Symposium on User Interface Software and Technology (UIST '94), Marina del Rey, CA, 1994.

[145] B. N. Schilit, G. Golovchinsky, and M. N. Price, "Beyond paper: Supporting active reading with free form digital ink annotations," in CHI '98: Proceedings of the SIGCHI Conference on Human Factors in Computing Systems, pp. 249-256, New York, NY, USA: ACM Press/Addison-Wesley Publishing Co., 1998.

[146] S. Schkolne, M. Pruett, and P. Schröder, "Surface drawing: Creating organic 3D shapes with the hand and tangible tools," in CHI '01: Proceedings of the SIGCHI Conference on Human Factors in Computing Systems, pp. 261-268, New York, NY, USA: ACM, 2001.

[147] D. A. Schon, The Reflective Practitioner. Basic Books, 1983.

[148] D. A. Schon and G. Wiggins, "Kinds of seeing and their functions in designing," Design Studies, vol. 13, no. 2, pp. 135-156, 1992.

[149] E. Schweikardt and M. D. Gross, "Digital clay: Deriving digital models from freehand sketches," in Digital Design Studios: Do Computers Make A Difference? ACADIA 98, (T. Seebohm and S. V. Wyk, eds.), pp. 202-211, 1998.

[150] T. Sezgin, T. Stahovich, and R. Davis, "Sketch based interfaces: Early processing for sketch understanding," in The Proceedings of 2001 Perceptive User Interfaces Workshop (PUI'01), 2001.

[151] T. M. Sezgin, "Sketch interpretation using multiscale stochastic models of temporal patterns," PhD thesis, Massachusetts Institute of Technology, 2006.

[152] T. M. Sezgin and R. Davis, "Sketch interpretation using multiscale models of temporal patterns," IEEE Computer Graphics and Applications, vol. 27, no. 1, pp. 28-37, 2007.

[153] M. Shilman, H. Pasula, S. Russell, and R. Newton, "Statistical visual language models for ink parsing," in AAAI Sketch Understanding Symposium, 2001.

[154] M. Shilman, Z. Wei, S. Raghupathy, P. Simard, and D. Jones, "Discerning structure from freeform handwritten notes," in Proceedings of International Conference on Document Analysis and Recognition (ICDAR) 2003, 2003. 
[155] M. Shpitalni and H. Lipson, "Classification of sketch strokes and corner detection using conic sections and adaptive clustering," Transactions of ASME Journal of Mechanical Design, vol. 119, no. 2, pp. 131-135, 1996.

[156] B. Signer and M. C. Norrie, "PaperPoint: A paper-based presentation and interactive paper prototyping tool," in TEI '07: Proceedings of the 1st International Conference on Tangible and Embedded Interaction, pp. 57-64, New York, NY, USA: ACM, 2007.

[157] H. A. Simon, "The structure of ill structured problems," Artificial Intelligence, vol. 4, no. 3, pp. 181-201, 1973.

[158] H. Song, F. Guimbretière, and H. Lipson, "ModelCraft: Capturing freehand annotations and edits on physical 3D models," in UIST '06: Proceedings of the 19th Annual ACM Symposium on User Interface Software and Technology, 2006.

[159] T. F. Stahovich, "Interpreting the engineer's sketch: A picture is worth a thousand constraints," in 1997 AAAI Symposium on Reasoning with Diagrammatic Representations II, pp. 31-38, 1997.

[160] M. Stefik, G. Foster, D. G. Bobrow, K. Kahn, S. Lanning, and L. Suchman, "Beyond the chalkboard: Computer support for collaboration and problem solving in meetings," Communications of the ACM, vol. 30, no. 1, pp. 32-47, 1987.

[161] I. Sutherland, "SketchPad: A man-machine graphical communication system," in Spring Joint Computer Conference, pp. 329-345, 1963.

[162] M. Suwa and B. Tversky, "What do architects and students perceive in their design sketches? A protocol analysis," Design Studies, vol. 18, pp. 385-403, 1997.

[163] M. Terry and E. D. Mynatt, "Recognizing creative needs in user interface design," in $C$ \& $C$ '02: Proceedings of the ACM Conference on Creativity and Cognition, 2002.

[164] L. Tesler, "The smalltalk environment," Byte, vol. 6, pp. 90-147, 1981.

[165] C. Thorpe and S. Shafer, "Correspondence in line drawings of multiple views of objects," in Proceedings of IJCAI-83, 1983.

[166] K. Tombre, C. Ah-Soon, P. Dosch, G. Masini, and S. Tabonne, "Stable and robust vectorization: How to make the right choices," in Graphics Recognition: Recent Advances, (A. Chhabra and D. Dori, eds.), vol. 1941 of Lecture Notes in Computer Science, Springer-Verlag, Berlin, pp. 3-18, 2000.

[167] S. Tsang, R. Balakrishnan, K. Singh, A. Ranjan, and A. Ranjan, "A suggestive interface for image guided 3D sketching," in CHI '04: Proceedings of the SIGCHI Conference on Human Factors in Computing Systems, pp. 591-598, New York, NY, USA: ACM, 2004.

[168] B. Tversky, "What do sketches say about thinking?," in AAAI Spring Symposium on Sketch Understanding, (T. Stahovic, J. Landay, and R. Davis, eds.), Menlo Park, CA: AAAI Press, 2002.

[169] B. Tversky and P. U. Lee, "Pictorial and verbal tools for conveying routes," in COSIT-99, (C. Freksa and D. M. Mark, eds.), Vol. 1661 of Lecture notes in Computer Science, Springer, Stade, Germany, pp. 51-64, 1999. 
[170] B. Tversky, J. Zacks, P. U. Lee, and J. Heiser, "Lines, blobs, crosses and arrows: Diagrammatic communication with schematic figures," in Diagrams '00: Proceedings of the First International Conference on Theory and Application of Diagrams, pp. 221-230, London, UK:, Springer-Verlag, 2000.

[171] P. van Sommers, Drawing and Cognition: Descriptive and Experimental Studies of Graphic Production Processes. Cambridge University Press, 1984.

[172] O. Veselova and R. Davis, "Perceptually based learning of shape descriptions," in AAAI '04: Proceedings of the National Conference on Artificial Intelligence, San Jose, California, pp. 482-487, 2004.

[173] Wacom, "Wacom tablet," http://www.wacom.com, 2007.

[174] M. Walker, L. Takayama, and J. A. Landay, "High-fidelity or low-fidelity, paper or computer? Choosing Attributes when testing web prototypes," in Proceedings of Human Factors and Ergonomics Society: HFES 2002, 2002.

[175] W. Wang and G. Grinstein, "A polyhedral object's CSG-Rep reconstruction from a single 2D line drawing," in Proceedings of 1989 SPIE Intelligent Robots and Computer Vision III: Algorithms and Techniques, pp. 230-238, 1989.

[176] J. R. Ward, "Annotated bibilography in pen computing and handwriting recognition," 2008. http://users.erols.com/rwservices/biblio.html.

[177] N. Wardrip-Fruin and N. Montfort, eds., The New Media Reader. MIT Press, 2003.

[178] D. West, A. Quigley, and J. Kay, "MEMENTO: A digital-physical scrapbook for memory sharing," Personal Ubiquitous Computers, vol. 11, no. 4, pp. 313$328,2007$.

[179] J. O. Wobbrock, A. D. Wilson, and Y. Li, "Gestures without libraries, toolkits or training: A $\$ 1$ recognizer for user interface prototypes," in UIST '07: Proceedings of ACM Symposium on User Interface Software and Technology, pp. 159-168, New York, NY, USA: ACM, 2007.

[180] Y. Y. Wong, "Rough and ready prototypes: Lessons from graphic design," in CHI '92: Posters and Short Talks of the 1992 SIGCHI Conference on Human Factors in Computing Systems, pp. 83-84, New York, NY, USA: ACM, 1992.

[181] Y. Yamamoto, K. Nakakoji, Y. Niahinaka, and M. Asada, "ART019: A timebased sketchbook interface," Technical report, KID Laboratory, RCAST, University of Tokyo.

[182] R. C. Zeleznik, K. P. Herndon, and J. F. Hughes, "SKETCH: An interface for sketching 3D scenes," in SIGGRAPH 1996, 1996. 